This material is posted here with permission of the IEEE. Such permission of the IEEE does not in any way imply IEEE endorsement of any of Helsinki University of Technology's products or services. Internal or personal use of this material is permitted. However, permission to reprint/republish this material for advertising or promotional purposes or for creating new collective works for resale or redistribution must be obtained from the IEEE by writing to pubs-permissions@ieee.org.

By choosing to view this document, you agree to all provisions of the copyright laws protecting it. 


\title{
Angular Power Distribution and Mean Effective Gain of Mobile Antenna in Different Propagation Environments
}

\author{
Kimmo Kalliola, Kati Sulonen, Heikki Laitinen, Outi Kivekäs, Joonas Krogerus, and Pertti Vainikainen, Member, IEEE
}

\begin{abstract}
We measured the elevation angle distribution and cross-polarization power ratio of the incident power at the mobile station in different radio propagation environments at $2.15-\mathbf{G H z}$ frequency. A novel measurement technique was utilized, based on a wide-band channel sounder and a spherical dual-polarized antenna array at the receiver. Data were collected over $9 \mathrm{~km}$ of continuous measurement routes, both indoor and outdoor. Our results show that in non-line-of-sight situations, the power distribution in elevation has a shape of a double-sided exponential function, with different slopes on the negative and positive sides of the peak. The slopes and the peak elevation angle depend on the environment and base-station antenna height. The cross-polarization power ratio varied within 6.6 and $11.4 \mathrm{~dB}$, being lowest for indoor and highest for urban microcell environments. We applied the experimental data for analysis of the mean effective gain (MEG) of several mobile handset antenna configurations, with and without the user's head. The obtained MEG values varied from approximately $-5 \mathrm{dBi}$ in free space to less than $-11 \mathrm{dBi}$ beside the head model. These values are considerably lower than what is typically used in system specifications. The result shows that considering only the maximum gain or total efficiency of the antenna is not enough to describe its performance in practical operating conditions. For most antennas, the environment type has little effect on the MEG, but clear differences exist between antennas. The effect of the user's head on the MEG depends on the antenna type and on which side of the head the user holds the handset.
\end{abstract}

Index Terms-Angular power distribution, mean effective gain, mobile antenna, polarization, radio channel sounding.

\section{INTRODUCTION}

$\mathbf{T}$ HE gain of a mobile handset antenna is a critical parameter in cellular network design. Due to the large variety of mobile phones used in networks, it is very important that their antenna performance be able to be evaluated reliably. The traditional definition of antenna gain is not adequate for evaluating

Manuscript received April 27, 2001; revised October 26, 2001. This work was supported by the National Technology Agency of Finland, by the Graduate School in Electronics, Telecommunications and Automation, by the Finnish Society of Electronics Engineers, by the HPY Foundation, by the Nokia Foundation, and by the Wihuri Foundation.

K. Kalliola was with Helsinki University of Technology, FIN-02015 HUT, Finland. He is now with Nokia Research Center, Radio Communications Laboratory, FIN-00045 Nokia Group, Finland (e-mail: kimmo.kalliola@nokia.com).

K. Sulonen, O. Kivekäs, and P. Vainikainen are with IDC/Radio Laboratory, Helsinki University of Technology, FIN-02015 HUT, Finland (e-mail kati.sulonen@hut.fi; outi.kivekas@hut.fi; pertti.vainikainen@hut.fi).

H. Laitinen is with VTT Information Technology, FIN-02044 VTT, Finland (e-mail: heikki.laitinen@vtt.fi)

J. Krogerus is with Nokia Research Center, Radio Communications Laboratory, FIN-00045 Nokia Group, Finland (e-mail: joonas.krogerus@nokia.com).

Digital Object Identifier 10.1109/TVT.2002.800639 the performance of a handset antenna, whose orientation relative to the direction and polarization of the incident field is unknown. Several methods have been proposed for determining the performance of a mobile antenna in realistic propagation conditions.

The random-field measurement (RFM) method [1]-[4] is based on measuring the mean received power level of the antenna on a random route in a typical operating environment. The mean effective gain (MEG) of the antenna is obtained as the ratio of the mean signal levels of the test antenna and a reference antenna. The effect of the user holding the handset on the antenna gain can be easily analyzed with this method [5], [6]. The method is naturally closest to reality, but it is time consuming, since the repeatability of the measurements is poor, and statistical significance can only be achieved by doing extensive measurements in all possible operating environments. The RFM method can be simplified by using a field simulator to produce an artificial scattering environment in an indoor facility [7], [8]. This makes the measurements repeatable, but it is not evident that the conditions resemble a realistic operating environment.

In [9], Taga derived a general expression for MEG. Using the formulas presented in [9], the MEG of an antenna in a certain environment can be computed based on the three-dimensional (3-D) gain pattern of the antenna and the average angular distribution of incident power in the environment. The power distribution must be known in both azimuth and elevation, and separately for horizontally $(\phi-)$ and vertically $(\theta-)$ polarized field components. Also, the cross-polarization power ratio (XPR) is needed in the calculation.

The clear benefit of the computational method for determining the MEG is that it is fast and repeatable. In addition to [9], it has been used in [4], [5], and [10]. Currently, the drawback is that there is little information available on realistic field distributions in different environments. From the random orientation of the mobile antenna in azimuth, it is straightforward to assume that the azimuth distribution of the waves is uniform. Instead, no straightforward assumption can be justified for the distribution in elevation. Few published results exist on measured elevation power distributions [9], [11], [12]. Only [9] proposes a parameterized model for the distribution and gives the model parameters fitted to experimental data at $900 \mathrm{MHz}$, but they are limited to an urban macrocell environment with large base-station antenna height. Another model was proposed in [13], but no measurement data were given to verify or tune the model. 
In this paper, we present experimental results of the elevation power distribution (EPD) and XPR at the mobile antenna in different radio environments at $2.15-\mathrm{GHz}$ frequency. The measurements were performed using a wide-band radio channel sounder and a spherical dual-polarized antenna array. The measurement method, described in [14], enables the full 3-D measurement of the spatial radio channel in real time, and thus the acquisition of statistically significant amounts of data. We compare two parameterized models for the EPD: the symmetrical Gaussian function proposed by Taga [9] and the asymmetrical general double-exponential function. We also present the fitted parameter values of both models for each measurement environment.

In addition, we apply the experimental results for MEG calculations of several practical mobile antenna configurations, with both measured and simulated radiation patterns. We consider the dependence of the MEG on the usage environment and compare the MEG of a handset antenna to the total efficiency, gain, and cross-polarization discrimination of the antenna configuration. Furthermore, we investigate the deviation of the MEG values caused by using the model instead of the measured EPDs.

\section{Mean Effective Gain}

According to Taga [9], the MEG of an antenna can be expressed using the 3-D power gain pattern of the antenna and the angular power density functions of radiowaves in a multipath environment, both defined separately for the $\theta$ - and $\phi$-polarized ${ }^{1}$ field components. Also, the cross-polarization power ratio, i.e., the ratio of the mean incident $\theta$ - and $\phi$-polarized powers along a random route in the case of $\theta$-polarized transmission [9], is needed in the computation of MEG. The angular power density functions $p_{\ominus}(\theta, \phi)$ and $p_{\phi}(\theta, \phi)$ need to satisfy the following condition:

$$
\begin{aligned}
\int_{0}^{2 \pi} \int_{-\pi / 2}^{\pi / 2} p_{\theta}(\theta, \phi) \cos \theta d \theta d \phi & \\
& =\int_{0}^{2 \pi} \int_{-\pi / 2}^{\pi / 2} p_{\phi}(\theta, \phi) \cos \theta d \theta d \phi \\
& =1 .
\end{aligned}
$$

The spherical coordinate system is defined in Fig. 1. It should be noted that according to (1), the definition of the angular power density functions differs from that of the joint probability density function for the direction of arrival $f(\theta, \phi)$, defined in [15], which satisfies

$$
\int_{0}^{2 \pi} \int_{-\pi / 2}^{\pi / 2} f(\theta, \phi) d \theta d \phi=1 .
$$

It follows by combining (1) and (2) that the relation between the angular power density function and joint probability density function for the direction of arrival is

$$
p(\theta, \phi)=\frac{f(\theta, \phi)}{\cos \theta} .
$$

It is natural to assume that when a mobile user moves randomly in any environment, the incident waves can arise from

\footnotetext{
${ }^{1} \theta$ and $\phi$ polarizations correspond to the vertical and horizontal polarizations used in [9] in the plane perpendicular to the incidence angle
}

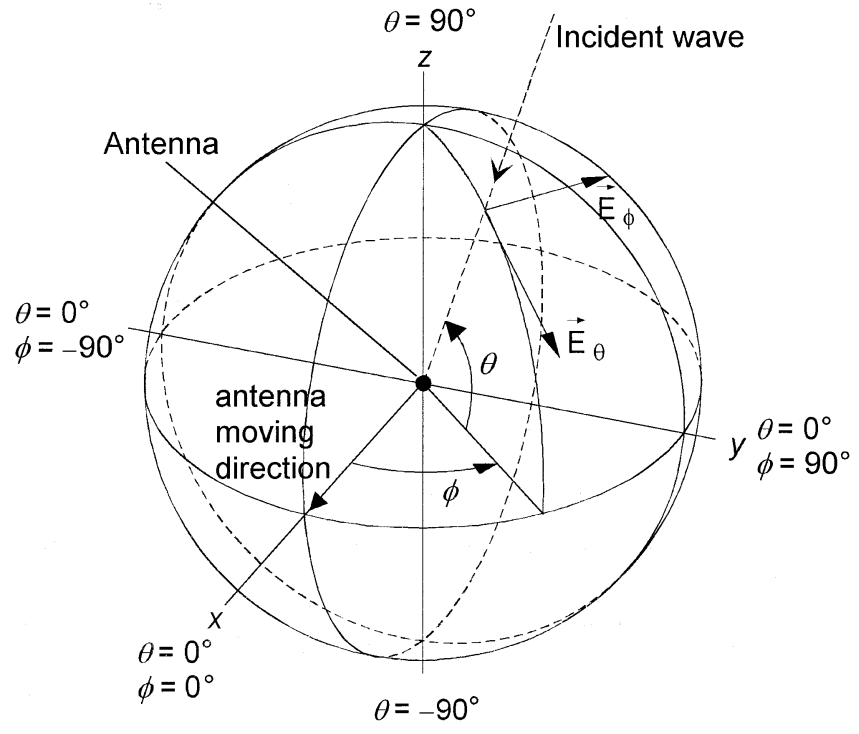

Fig. 1. Spherical coordinate system.

any azimuth direction with equal probability. If the power density function in azimuth is uniform and independent of elevation, the joint angular power density functions reduce to

$$
p(\theta, \phi)=\frac{1}{2 \pi} p(\theta) .
$$

In contrast to the azimuth, no straightforward assumption can be justified for the power distribution in elevation. Previously, few models have been presented. The first model by Clarke [16] assumes that all energy is concentrated in the horizontal plane. However, it is known to be unrealistic at least in urban environments, where buildings give rise to multipath components from high elevation angles. Aulin [17] proposed a generalization of Clarke's model to a case where all energy does not travel in the horizontal plane, but no data were available to verify the model. Also, [13], which proposes a family of functions to describe the power distribution in elevation, lacks measurement data. In [9], Taga proposed the Gaussian density function and fitted the parameters to experimental data. However, he used only four measured points of the elevation power distribution, which is not necessarily sufficient to verify the distribution.

\section{DESCRIPTION OF EXPERIMENTS}

\section{A. Measurement Setup}

We measured the angular power distribution separately for $\theta$ and $\phi$-polarized components of the incident field at the mobile station in different propagation environments using the measurement method presented in [14]. The method is based on a spherical array of 32 dual-polarized antenna elements and a complex wide-band radio channel sounder. At the base station (BS), a wide-band signal was transmitted using a single fixed vertically polarized antenna. At the mobile station (MS), the signal was received separately from the $\theta$ - and $\phi$-polarized feeds of each of the 32 elements of the spherical array, using a fast 64-channel radio-frequency switch. Approximately five snapshots $^{2}$ of the received signal were sampled and stored per each

\footnotetext{
${ }^{2}$ One PN sequence period from all 64 channels.
} 
TABLE I

Definitions of ANGUlar Power Didistributions. Subscripts Denote Polarizations

\begin{tabular}{|c|c|c|}
\hline Definition & Azimuth & Elevation \\
\hline $\begin{array}{l}\text { Instantaneous power as } \\
\text { a function of incidence } \\
\text { angle. }\end{array}$ & $\begin{array}{l}P_{\theta, i}(\phi)=\left.\left.\iint_{\tau}\right|_{\theta} h_{\theta, i}(\theta, \phi, \tau)\right|^{2} \cos \theta d \theta d \tau \\
P_{\phi, i}(\phi)=\left.\iint_{\tau} \int_{\theta} h_{\phi, i}(\theta, \phi, \tau)\right|^{2} \cos \theta d \theta d \tau\end{array}$ & $\begin{array}{l}P_{\theta, i}(\theta)=\iint_{\tau}\left|h_{\theta, i}(\theta, \phi, \tau)\right|^{2} d \phi d \tau \\
P_{\phi, i}(\theta)=\iint_{\tau}\left|h_{\phi}(\theta, \phi, \tau)\right|^{2} d \phi d \tau\end{array}$ \\
\hline $\begin{array}{l}\text { Mean relative power as } \\
\text { a function of incidence } \\
\text { angle. }\end{array}$ & $\begin{array}{l}P_{\theta}(\phi)=\frac{1}{N} \sum_{i=1}^{N} \frac{P_{\theta, i}(\phi)}{\oint\left[P_{\theta, i}(\phi)+P_{\phi, i}(\phi)\right] d \phi} \\
P_{\phi}(\phi)=\frac{1}{N} \sum_{i=1}^{N} \frac{P_{\phi, i}(\phi)}{\oint\left[P_{\theta, i}(\phi)+P_{\phi, i}(\phi)\right] d \phi}\end{array}$ & $\begin{array}{l}P_{\theta}(\theta)=\frac{1}{N} \sum_{i=1}^{N} \frac{P_{\theta, i}(\theta)}{\oint\left[P_{\theta, i}(\theta)+P_{\phi, i}(\theta)\right] \cos \theta d \theta} \\
P_{\phi}(\theta)=\frac{1}{N} \sum_{i=1}^{N} \frac{P_{\phi, i}(\theta)}{\oint\left[P_{\theta, i}(\theta)+P_{\phi, i}(\theta)\right] \cos \theta d \theta}\end{array}$ \\
\hline $\begin{array}{l}\text { Power distributions in } \\
\text { azimuth (APD) and } \\
\text { elevation (EPD). }\end{array}$ & $\begin{array}{l}p_{\theta}(\phi)=\frac{P_{\theta}(\phi)}{\oint P_{\theta}(\phi) d \phi} \\
p_{\phi}(\phi)=\frac{P_{\phi}(\phi)}{\oint P_{\phi}(\phi) d \phi}\end{array}$ & $\begin{array}{l}p_{\theta}(\theta)=\frac{P_{\theta}(\theta)}{\oint P_{\theta}(\theta) \cos \theta d \theta} \\
p_{\phi}(\theta)=\frac{P_{\phi}(\theta)}{\oint P_{\phi}(\theta) \cos \theta d \theta}\end{array}$ \\
\hline
\end{tabular}

wavelength the mobile moved, except for the highway macrocell environment, where the number of snapshots per wavelength was between two and three. The center frequency was $2.154 \mathrm{GHz}$ and the carrier was modulated by a PN-sequence with $30-\mathrm{MHz}$ chip frequency. Detailed information of the used Helsinki University of Technology, Institute of Digital Communications (IDC) channel sounder can be found in [18].

During the measurements, the transmitting antenna was placed in fixed locations corresponding to typical BS antenna installations in different cellular radio network configurations. A modified commercial GSM1800 sector antenna with $10-\mathrm{dBi}$ gain and $3-\mathrm{dB}$ beamwidth of $80^{\circ}$ in azimuth and $28^{\circ}$ in elevation was used in all cases except for the indoor picocell, where the transmitting antenna was omnidirectional (vertical 3 -dB beamwidth $80^{\circ}$, gain $2 \mathrm{dBi}$ ). It must be noted that the radiation pattern of the transmitting antenna affects the signal distribution at the receiver. The transmitting antennas were chosen as typical examples of BS antennas used in existing networks at cell configurations similar to the measured ones to obtain as realistic results as possible.

\section{B. Processing of Data}

The delays, directions of arrival (DoAs), amplitudes, and phases of both $\theta$ - and $\phi$-polarized components of the incoming waves at each measurement snapshot were found through sequential delay-domain and angular-domain processing. First, the received signal of each antenna feed was correlated with a replica of the transmitted PN-sequence to obtain the complex impulse response of the channel. The delay taps were then identified by detecting the local maxima of the power-delay profile averaged over the array elements. Corresponding to each delay tap, there may exist one multipath component or several components separated by their DoAs. Up to four multipath components per delay tap were estimated using the beamforming scheme with precomputed array weights $\left(2^{\circ}\right.$ beam spacing in azimuth and elevation), as described in [14]. Only such multipaths were accepted whose amplitude exceeded a threshold value of $6 \mathrm{~dB}$ below the highest multipath, in order to filter out spurious signals due to sidelobes of the array. The measured sidelobe level of the array is approximately $-10 \mathrm{~dB}$ in the case of two simultaneous multipaths [14], but the level increases with an increasing number of multipaths.

The amplitudes and phases of the $\theta$ - and $\phi$-polarized components of the incident waves were obtained by pointing $\theta$ - and $\phi$-polarized beams in these directions. As a final result, we had for each measurement snapshot the angle resolved impulse response, defined as

$$
\left[\begin{array}{l}
h_{\ominus}(\theta, \phi, \tau) \\
h_{\phi}(\theta, \phi, \tau)
\end{array}\right]=\sum_{l=1}^{L}\left[\begin{array}{l}
\alpha_{\ominus, l} \\
\alpha_{\phi, l}
\end{array}\right] \delta\left(\theta-\theta_{l}\right) \delta\left(\phi-\phi_{l}\right) \delta\left(\tau-\tau_{l}\right)
$$

where $h_{\bullet}$ and $h_{\phi}$ denote the $\theta$ - and $\phi$-polarized components of the impulse response, respectively. $\alpha_{\ominus, l}$ and $\alpha_{\phi, l}$ are the complex amplitudes of $l^{t h}$ multipath, $\theta_{l}$ and $\phi_{l}$ are the corresponding elevation and azimuth angles, and $\tau_{l}$ is the delay.

The total spatial resolution of the measurement is determined by the angular resolution of the spherical array of approximately $40^{\circ}$ and the 33-ns delay resolution of the wide-band channel sounder. Thus the spatially separable blocks are truncated cones with an opening angle of $40^{\circ}$ and length of $10 \mathrm{~m}$, and the size of the block increases with increasing distance between the last scattering point and the array. The azimuth and elevation power distributions for $\theta$ - and $\phi$-polarized wave components were derived from the angle resolved impulse response using formulas presented in Table I. First the instantaneous power versus incidence angle was computed as a sum of the multipath powers. Then the mean relative power versus incidence angle in one environment was obtained by averaging over all snapshots in the environment. The variation of the received power due to large-scale fading was compensated by normalizing the result to 
TABLE II

AMOUNT OF COLLECTED DATA

\begin{tabular}{|c|c|c|c|c|}
\hline Environment & Route length & Snapshots & LOS & $N L O S$ \\
\hline Indoor picocell (Airport) & $260 \mathrm{~m}$ & $N=9800$ & $40 \%$ & $60 \%$ \\
\hline Outdoor - Indoor (Office) & $220 \mathrm{~m}$ & $N=8300$ & $0 \%$ & $100 \%$ \\
\hline $\begin{array}{l}\text { Urban microcell } \\
\left(\mathrm{h}_{\mathrm{BS}}=3 / 8 / 13 \mathrm{~m}\right)\end{array}$ & $\begin{array}{l}3 \times 1200 \mathrm{~m} \\
=3600 \mathrm{~m}\end{array}$ & $\begin{array}{l}3 \times 51000 \\
N=153000\end{array}$ & $22 \%$ & $78 \%$ \\
\hline $\begin{array}{l}\text { Urban macrocell } \\
\left(\mathrm{h}_{\mathrm{BS}}=21 / 27 \mathrm{~m}\right)\end{array}$ & $2560 \mathrm{~m}$ & $N=100600$ & $4 \%$ & $96 \%$ \\
\hline Highway macrocell & $2500 \mathrm{~m}$ & $N=47600$ & $25+45^{*} \%$ & $30 \%$ \\
\hline TOTAL & $9140 \mathrm{~m}$ & $N=319300$ & $23 \%$ & $77 \%$ \\
\hline
\end{tabular}

LOS obstructed by trees

the total incident power at each point. Due to the large measurement bandwidth compared to the channel coherence bandwidth, the fast fading of the total received power (sum of the multipath powers) was small. The distribution of the square root of the total power agreed well with Rice distribution. The average fitted Rice factor varied from $k \approx 17$ in the outdoor-indoor case to $k \approx 60$ in the urban macrocell case. Finally, the azimuth and elevation power distributions (APDs and EPDs, respectively) were obtained from the mean relative powers versus incidence angle as presented in Table I.

\section{Measurement Environments}

We performed measurements in five different radio environments. The environments, the approximate total route lengths, and the numbers of collected snapshots are presented in Table II. Also, the percentages of line-of-sight (LOS) and non-line-ofsight (NLOS) measurements are shown. The characteristic features of each environment are briefly described below. In all measurements except those of the highway macrocell, the spherical array acting as the mobile station was mounted on a trolley, where the center of the array was at a height of $1.7 \mathrm{~m}$ above ground level and the visible arc in elevation was from zenith to approximately $-60^{\circ}$.

The indoor picocell measurements were carried out in the transit hall of Helsinki airport. The omnidirectional BS antenna was elevated at $4.6 \mathrm{~m}$ above the floor level and located so that the visibility over the hall was good. The BS-MS distance varied from 10 to $150 \mathrm{~m}$. The portion of LOS measurements was significant, on the order of $40 \%$.

The outdoor-indoor measurements were performed in two different office buildings, both having four floors and office rooms next to the outer walls made of brick. In both sites, the BS antenna was placed on the rooftop of the neighboring building. The average distance from the BS to the mobile routes was in the range of 50-100 $\mathrm{m}$. The short distance was forced by the limited sensitivity of the measurement due to the losses in the switching unit. The BS antenna was approximately 3 and $8 \mathrm{~m}$ above the mobile antenna for the two sites, respectively. The measurement routes include both corridors and office rooms, and the ceiling height is in the range of $2.5-3 \mathrm{~m}$ in both buildings.

The urban micro- and macrocell measurements were performed in the center of Helsinki, Finland. The spherical array was located on a trolley, and the routes were driven along the sidewalks of the streets. Fig. 2 presents a map showing the BS positions and the measured mobile routes. The street grid in the measurement area is fairly regular, and the average street width is approximately $15 \mathrm{~m}$. The height of most buildings is in the range of $20-30 \mathrm{~m}$.

In the microcell environment, the same mobile routes were measured for the same BS location with three different BS antenna heights. The antenna was located on the sidewalk of a street and mounted on a person lift elevated at 3, 8, and $13 \mathrm{~m}$ above the street level. The main beam of the antenna pointed west along the street. The measurement routes included the main street with LOS to the BS, the two parallel streets on both sides, and four transversal streets in front of the antenna (see Fig. 2). The BS-MS distance varied from 10 to $350 \mathrm{~m}$.

In the urban macrocell measurements, the BS antenna was located on the rooftop of a building and pointed separately to two opposite directions in order to cover a larger area (see Fig. 2). The antenna heights from ground were 27 and $21 \mathrm{~m}$, the former being at, and the latter above, the rooftop level of the opposite buildings. Photographs showing the views from both macrocell antenna installations can be found in [19]. The BS-MS distance varied from 50 to $750 \mathrm{~m}$. Due to the limited sensitivity of the measurement system, we were not able to measure propagation distances corresponding to the radii of the biggest urban macrocells. However, our measurement distances are in line with many current urban site configurations in cellular networks today.

The highway macrocell measurements were carried out in an industrial area in Espoo, Finland. The BS antenna was located on top of a building next to a junction of a ring road with a lot 


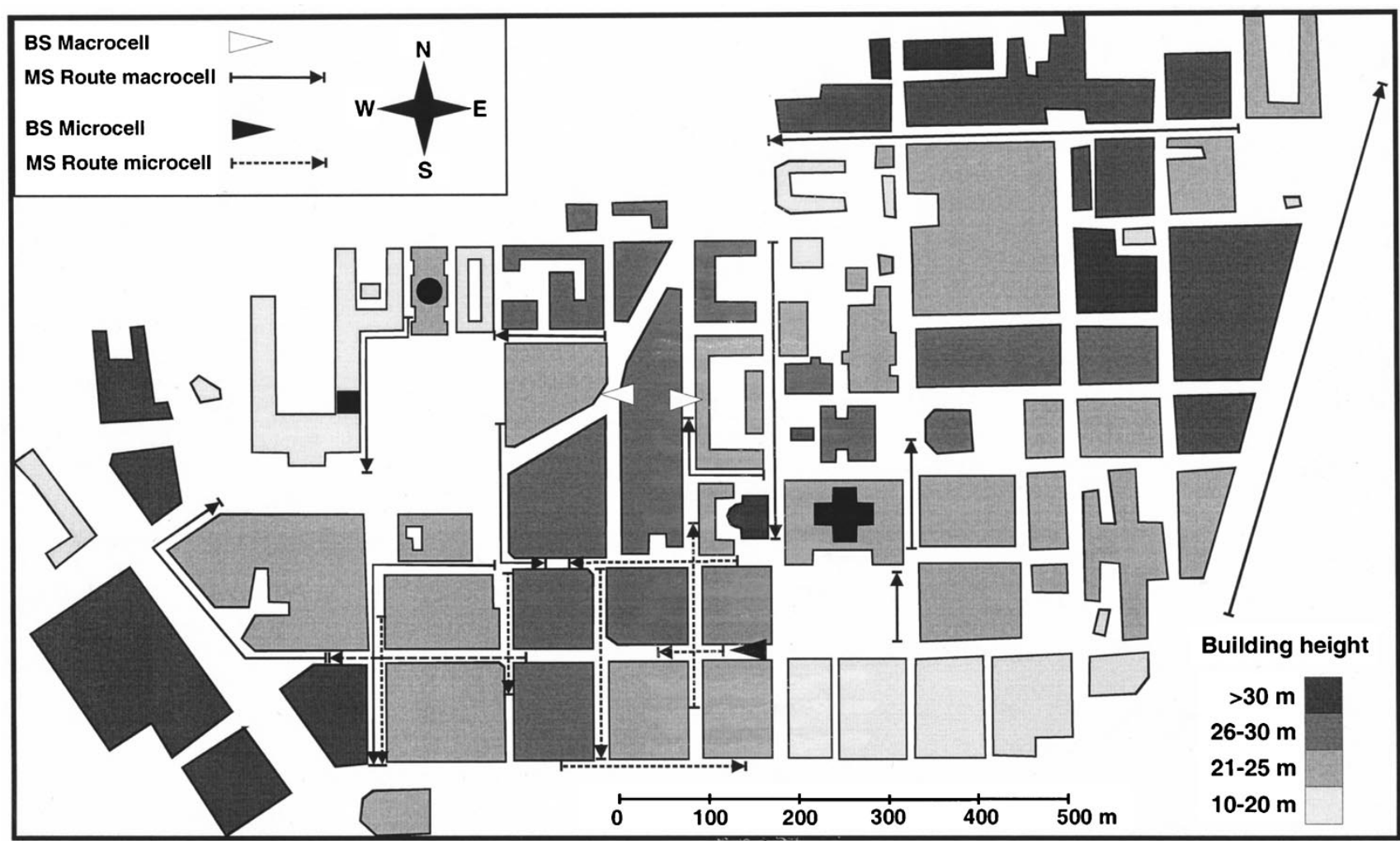

Fig. 2. Measurement routes in the center of Helsinki.

of traffic. Few large buildings exist in the area, and for most of the routes only trees obstruct the direct LOS path. The BS antenna height was $17 \mathrm{~m}$. The measurement routes included the transversal ring road and the crossing road. The BS-MS distance varied from 50 to $1200 \mathrm{~m}$. In these measurements, the spherical array was mounted inside a person car, on the front seat, and at the height of the passenger's head $(1.3 \mathrm{~m})$. It must be noted that the metallic chassis of the car has a significant effect on the angular distribution and polarization of the electric field at the antenna inside the car. However, it is common nowadays to use mobile handsets inside vehicles, which makes this a practical scenario.

\section{Statistical Distribution OF INCIDENT WAVES}

\section{A. Experimental Azimuth Power Distribution}

When a mobile antenna moves along a random route, uniform distribution is the only reasonable assumption for the power distribution in azimuth. However, to illustrate the radio propagation in urban environment, we present two examples of measured power distributions in different environments. Fig. 3 shows the APD in urban microcell $\left(h_{\mathrm{BS}}=3 \mathrm{~m}\right)$ and urban macrocell environments. It can be seen that the distributions are not at all uniform, but that some directions are more probable than others. This is due to the fact that the measured mobile routes are not random in nature but are parallel and perpendicular to street canyons (see Fig. 2). In the microcell case [Fig. 3(a)], the highest maxima are produced in directions close to $0^{\circ}$ and $180^{\circ}$, i.e., the directions of the street canyon along which the mobile is moving. The third maximum at left $\left(90^{\circ}\right)$ is due to propagation via crossing streets. The routes were by chance chosen so that the BS is almost always at the left-hand side when looking to the moving direction of the mobile. If this had not been the case, the obtained azimuth power distribution would have four maxima with angular separation of $90^{\circ}$. In the macrocell case [Fig. 3(b)], the APD is closer to uniform than in the microcell, but the left-hand side of the distribution (angles $0^{\circ} \cdots+180^{\circ}$ ) clearly dominates. As in the microcell, the reason for this is that the routes were not random. However, since a real mobile user may turn around or cross the streets at any angle, uniform distribution is the only justified assumption for the azimuth power distribution averaged over a random route in any environment. Fig. 3 shows that in both urban micro- and macrocell cases, the differences between the $\theta$ - and $\phi$-polarized distributions are small.

\section{B. Experimental Elevation Power Distribution}

In contrast to the azimuth, it seems obvious that the elevation power distribution depends on the environment type as well as on the BS antenna height and BS-MS distance. Fig. 4 presents the mean relative power versus elevation angle in different environments. Also, the average of all distributions is presented. The figure demonstrates that the EPD depends on the environment and the BS antenna height. In all environments dominated by NLOS channels, the shape of the EPD is similar for angles close to horizontal plane: the power decays roughly exponentially on both sides of the peak of the EPD, until approximately $1 \%$ of the peak power. Both the slope of the exponential decay 


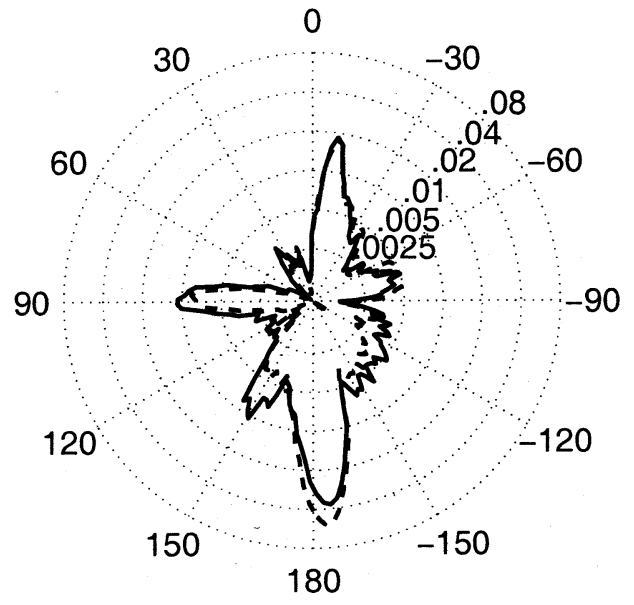

(a)

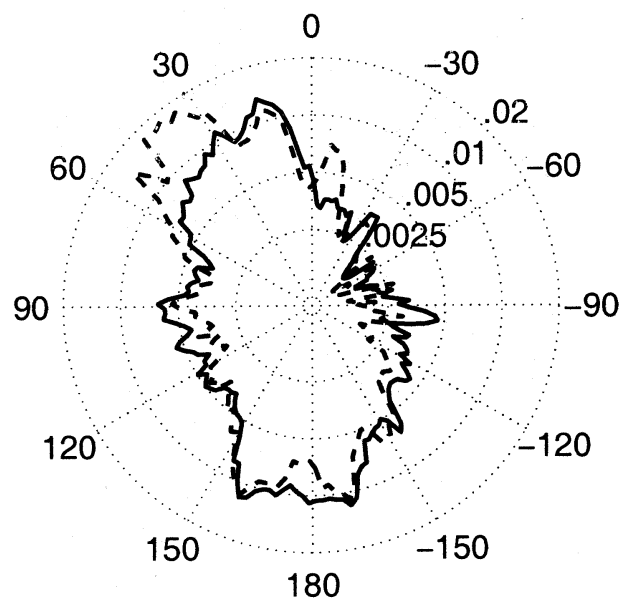

(b)

Fig. 3. Mean azimuth power distributions. (a) Urban microcell, $h_{\mathrm{BS}}=3 \mathrm{~m}$. (b) Urban macrocell. Mobile moving direction is $0^{\circ}\left(-P_{\ominus}(\phi),---P_{\phi}(\phi)\right)$.

and the shape of the EPD outside the main lobe depend on the environment. It should be noted, though, that in all environments, most of the power is concentrated in small positive elevation angles. This is in agreement with measurements by Lee and Brandt [20], who showed that most of the power is concentrated in elevation angles lower than $16^{\circ}$ above horizontal level.

In the indoor picocell measurements, a significant portion of the mobile routes contains LOS, which can be seen in the $\theta$-polarized EPD in Fig. 4(a) as peaks in the angle range of $10-20^{\circ}$ above horizontal plane. The LOS components with high elevation angles make the main lobe of the distribution wider also in the highway macrocell measurements. In the urban outdoor case, the EPD becomes asymmetrical and more power is received at high elevation angles when the BS antenna is raised above the rooftop level. The negative slope of the EPD changes hardly at all. For large antenna heights, also the XPR decreases for increasing elevation angle. In the urban macrocell case, the XPR is approximately $0 \mathrm{~dB}$ for elevation angles above $60^{\circ}$. In the indoor picocell and outdoor-indoor cases, the received power outside the main lobe is notably higher than in the outdoor measurements, most likely due to reflections from the ceiling. Similarly, the effect of the car can be seen as distortions in the EPD of the highway macrocell.
The elevation power distribution is described by the median elevation angle $\left(M_{\theta}\right)$, mean elevation angle $(\bar{\theta})$, and rms elevation spread $\left(\sigma_{\theta}\right)$, defined as

$$
\begin{aligned}
M_{\theta} \text {, so that } \int_{-\pi / 2}^{M_{\theta}} p(\theta) \cos \theta d \theta & =\frac{1}{2} \\
\bar{\theta} & =\int_{-\pi / 2}^{\pi / 2} \theta p(\theta) \cos \theta d \theta \\
\sigma_{\theta} & =\int_{-\pi / 2}^{\pi / 2}[\theta-\bar{\theta}]^{2} p(\theta) \cos \theta d \theta
\end{aligned}
$$

where $p(\theta)$ is either $p_{\Theta}(\theta)$ or $p_{\phi}(\theta)$. The parameters of the measured EPDs of all environments are presented in Table III, from which the following observations can be made.

1) The rms elevation angle spread is larger for horizontal polarization in all environments.

2) In all outdoor environments, the mean elevation angle is higher for horizontal polarization. In the indoor picocell, the situation is the opposite, due to the large portion of LOS measurements.

3) In all environments, the mean elevation angle is higher than the median, i.e., the distribution is asymmetrical so that the spread is larger for high elevation angles than for low angles.

4) In the urban environment, the mean and median elevation angle, as well as the elevation spread, increase when the BS antenna height increases. A clear step in mean values can be seen between BS antenna heights of 8 and $13 \mathrm{~m}$ in the urban microcell case.

\section{Model for Distribution in Elevation}

It is proposed in [9] that the EPD has Gaussian shape in an urban environment when no LOS exists between the MS and BS. However, as was observed in the previous section, the distribution is often not symmetrical about its peak value but decreases more rapidly on the negative side. Particularly, this seems to be the case for BS antenna heights larger than $10 \mathrm{~m}$, which is common for outdoor base stations also in urban environments. Fig. 5 presents the measured EPD for $\theta$-polarization in an urban macrocell environment together with two best fit distribution functions.

1) Gaussian function:

$$
p(\theta)=A_{1} \exp \left[-\frac{\left(\theta-\theta_{0}\right)^{2}}{2 \sigma^{2}}\right], \quad \theta \in\left[-\frac{\pi}{2}, \frac{\pi}{2}\right] .
$$

2) General double exponential function ${ }^{3}$ :

$$
p(\theta)= \begin{cases}A_{2} \exp \left[-\frac{\sqrt{2}\left|\theta-\theta_{0}\right|}{\sigma^{-}}\right], & \theta \in\left[-\frac{\pi}{2}, \theta_{0}\right] \\ A_{2} \exp \left[-\frac{\sqrt{2}\left|\theta-\theta_{0}\right|}{\sigma^{+}}\right], & \theta \in\left[\theta_{0}, \frac{\pi}{2}\right] .\end{cases}
$$

In (9) and (10), $p(\theta)$ is either $p_{\Theta}(\theta)$ or $p_{\phi}(\theta), \theta_{0}$ is the peak elevation angle, and parameters $\sigma, \sigma^{-}$, and $\sigma^{+}$control the spread of the functions. Coefficients $A_{1}$ and $A_{2}$ are set so that (1) is satisfied.

\footnotetext{
${ }^{3}$ If the spread parameters $\sigma^{-}$and $\sigma^{+}$are equal, the double exponential function is called Laplacian function.
} 


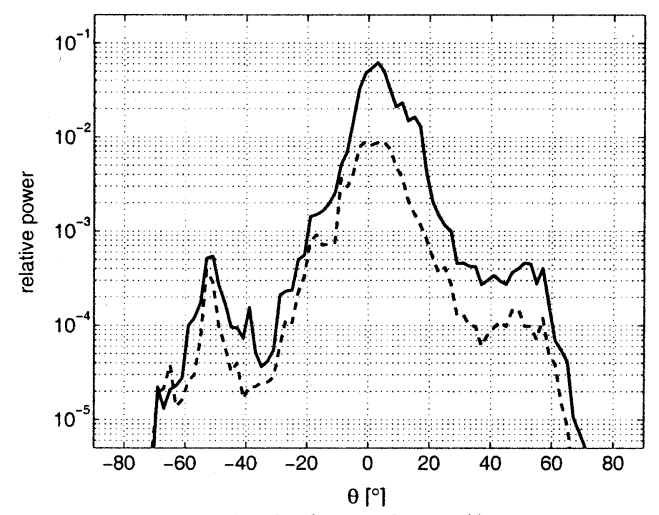

(a) indoor picocell

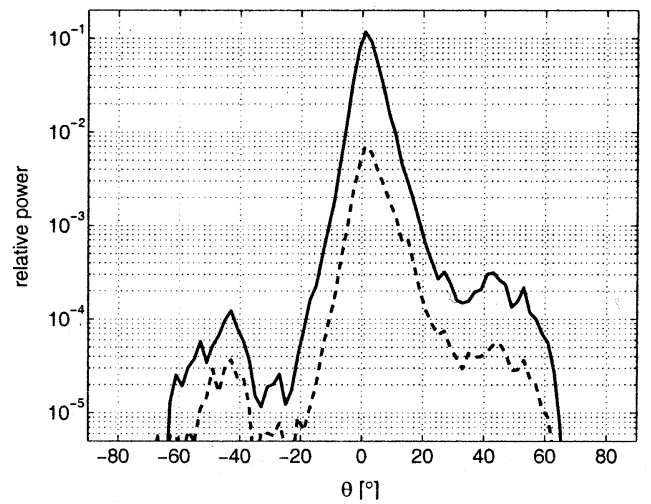

(c) urban microcell, $\mathrm{h}_{\mathrm{BS}}=3 \mathrm{~m}$

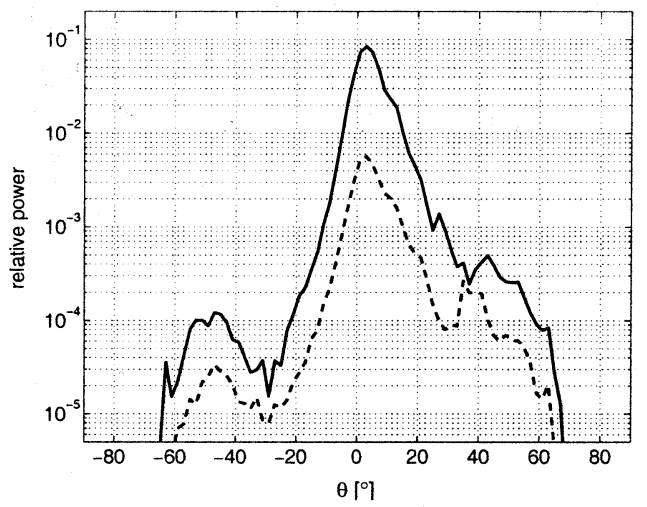

(e) urban microcell, $\mathrm{h}_{\mathrm{BS}}=13 \mathrm{~m}$

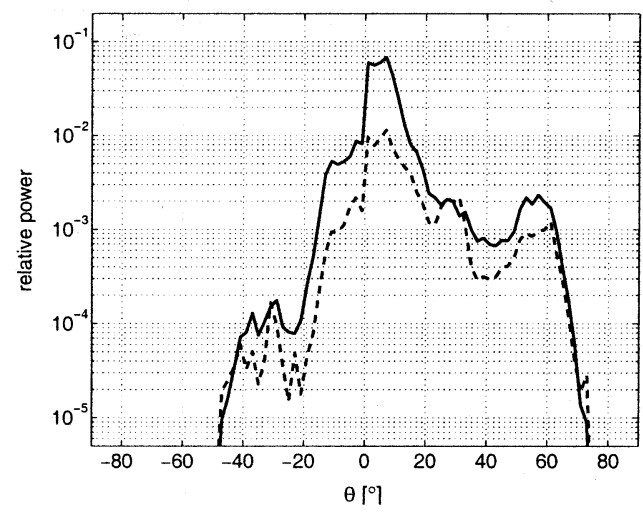

(g) highway macrocell

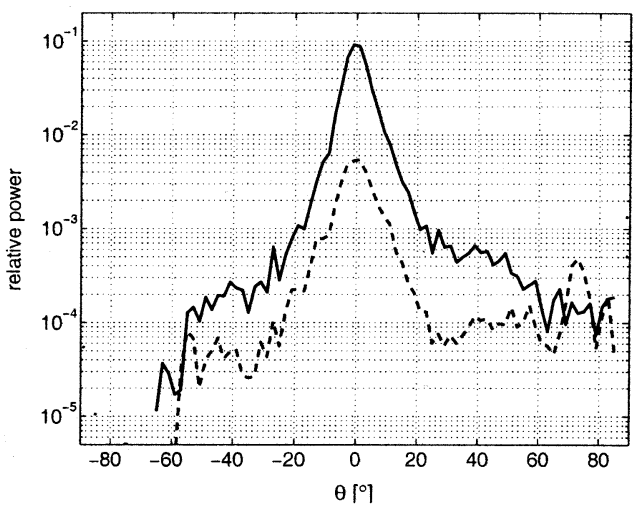

(b) outdoor - indoor

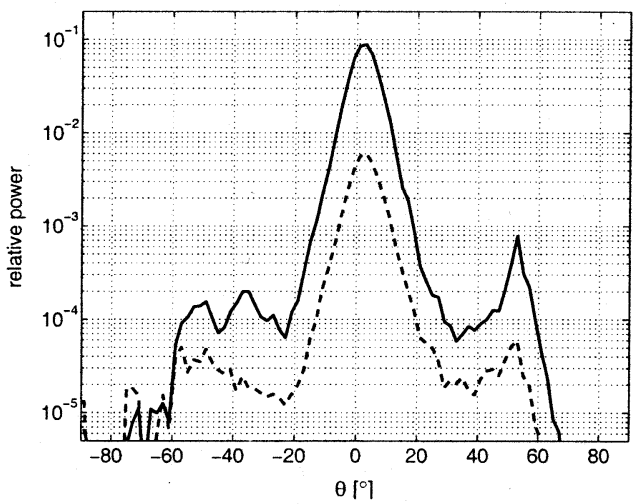

(d) urban microcell, $\mathrm{h}_{\mathrm{BS}}=8 \mathrm{~m}$

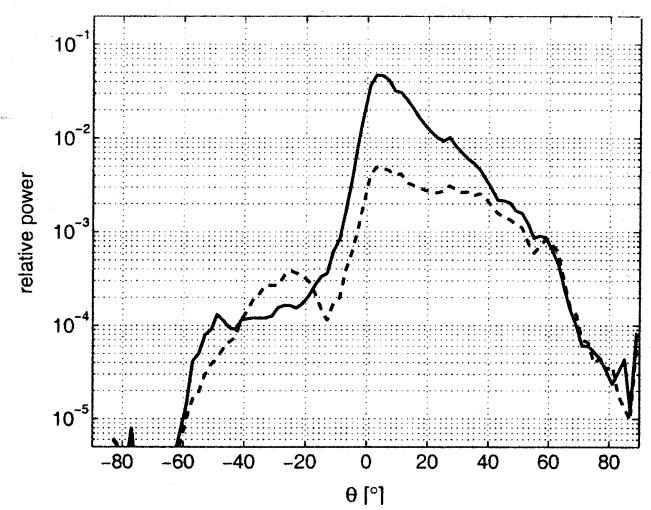

(f) urban macrocell

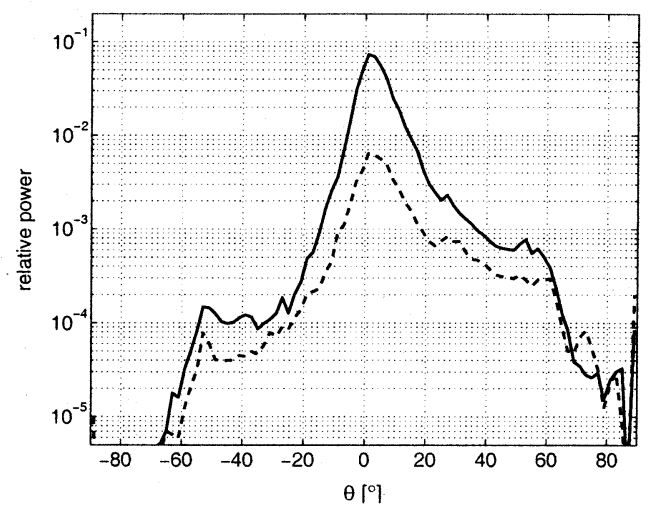

(h) average of environments

Fig. 4. Mean relative power versus elevation angle at mobile station in different radio environments $\left(-P_{\odot}(\theta),---P_{\phi}(\phi)\right)$. 
TABLE III

EPD PARAMETERS $\left(P_{\ominus} / P_{\phi}\right)$ AND XPR

\begin{tabular}{|c|c|c|c|c|}
\hline Environment & $M_{\theta}$ & $\bar{\theta}$ & $\sigma_{\theta}$ & $X P R$ \\
\hline Indoor picocell & $2.1^{\circ} / 1.1^{\circ}$ & $3.8^{\circ} / 1.9^{\circ}$ & $8.7^{\circ} / 10.7^{\circ}$ & $7.0 \mathrm{~dB}$ \\
\hline Outdoor - Indoor & $-1.0^{\circ} /-0.9^{\circ}$ & $0.6^{\circ} / 1.8^{\circ}$ & $8.1^{\circ} / 14.6^{\circ}$ & $10.7 \mathrm{~dB}$ \\
\hline \multicolumn{5}{|l|}{ Urban microcell: } \\
\hline $\mathrm{h}_{\mathrm{BS}}=3 \mathrm{~m}$ & $0.7^{\circ} / 1.5^{\circ}$ & $2.2^{\circ} / 3.5^{\circ}$ & $5.4^{\circ} / 7.8^{\circ}$ & $11.4 \mathrm{~dB}$ \\
\hline $\mathrm{h}_{\mathrm{BS}}=8 \mathrm{~m}$ & $1.3^{\circ} / 1.6^{\circ}$ & $2.4^{\circ} / 2.6^{\circ}$ & $6.2^{\circ} / 8.5^{\circ}$ & $11.4 \mathrm{~dB}$ \\
\hline $\mathrm{h}_{\mathrm{BS}}=13 \mathrm{~m}$ & $2.7^{\circ} / 3.1^{\circ}$ & $4.6^{\circ} / 5.7^{\circ}$ & $6.9^{\circ} / 9.9^{\circ}$ & $11.1 \mathrm{~dB}$ \\
\hline Urban macrocell & $7.2^{\circ} / 13.8^{\circ}$ & $11.2^{\circ} / 17.1^{\circ}$ & $11.9^{\circ} / 18.5^{\circ}$ & $7.3 \mathrm{~dB}$ \\
\hline Highway macrocell & $4.5^{\circ} / 6.4^{\circ}$ & $6.6^{\circ} / 10.9^{\circ}$ & $10.4^{\circ} / 14.5^{\circ}$ & $6.6 \mathrm{~dB}$ \\
\hline $\begin{array}{l}\text { Average of all } \\
\text { environments }\end{array}$ & $2.0^{\circ} / 3.7^{\circ}$ & $4.4^{\circ} / 7.5^{\circ}$ & $9.0^{\circ} / 14.7^{\circ}$ & $9.0 \mathrm{~dB}$ \\
\hline
\end{tabular}

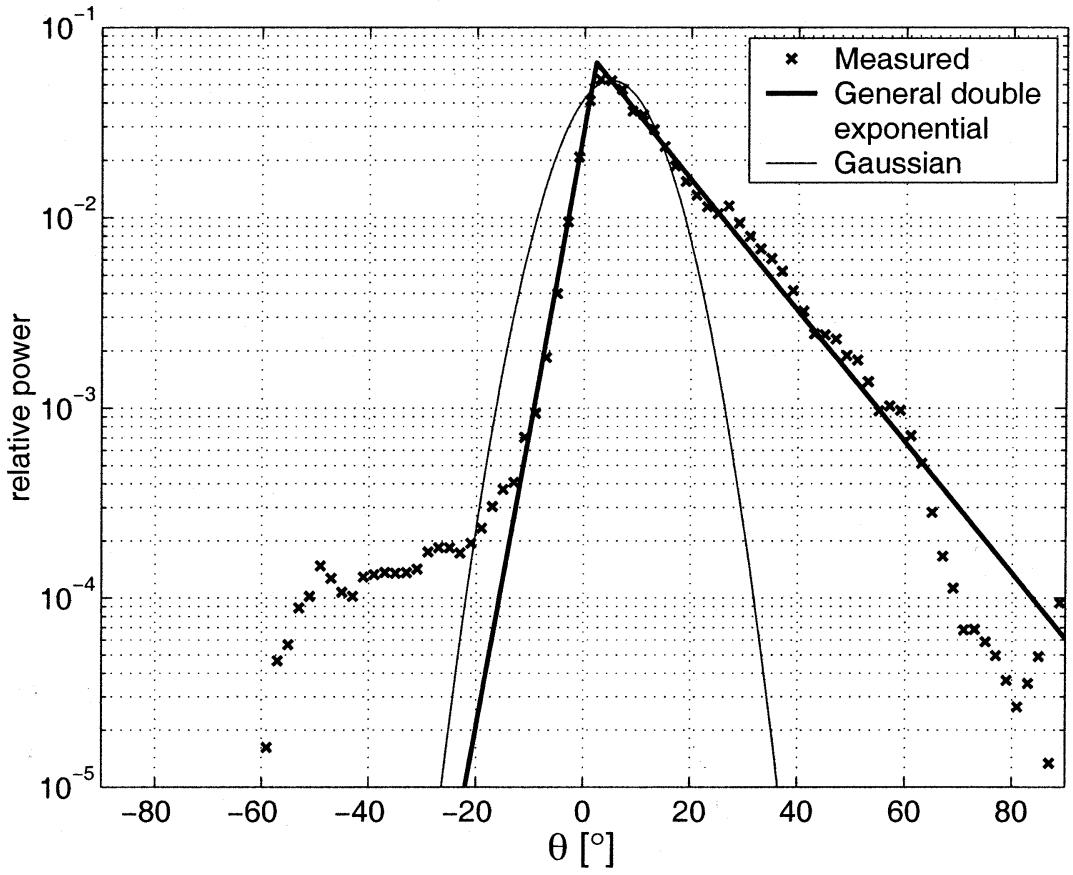

Fig. 5. Example of two EPD models. Measurement data from urban macrocell. $\theta$ polarization.

As can be seen in Fig. 5, in the case of an asymmetrical $\mathrm{EPD}$, the fitted symmetrical Gaussian function $\left(\theta_{0}=5.0^{\circ}\right.$, $\left.\sigma=7.6^{\circ}\right)$ does not give an accurate description of the EPD. Instead, the fitted general double exponential function $\left(\theta_{0}=2.2^{\circ}\right.$, $\sigma^{-}=3.9^{\circ}, \sigma^{+}=17.8^{\circ}$ ) gives an accurate match with measured data for values larger than $0.7 \%$ of the peak value. To find a representative statistical model for the elevation power distribution, and to study the effect of the model on the obtained mean effective gain of a mobile antenna, we fitted the measured power distributions in each environment to both model func- tions by minimizing the squared approximation error (SAE) of the fitted curves, defined as

$$
\mathrm{SAE}=\int_{-\pi / 2}^{\pi / 2}\left[p_{\text {fit }}(\theta)-p_{\text {meas }}(\theta)\right]^{2} d \theta
$$

where $p_{\text {fit }}$ and $p_{\text {meas }}$ are the modeled and measured distributions, respectively. Table IV presents the best fit parameters and approximation error of both functions. It can be seen that for most environments the SAE of the double exponential function is smaller than that of the Gaussian function, which indicates 
TABLE IV

Best-Fit Parameters of EPD Models $\left(P_{\ominus} / P_{\phi}\right)$

\begin{tabular}{|c|c|c|c|c|c|c|c|}
\hline \multirow[b]{2}{*}{ Environment } & \multicolumn{3}{|c|}{ Gaussian function } & \multicolumn{4}{|c|}{ General double exponential function } \\
\hline & $\theta_{0}\left[^{\circ}\right]$ & $\sigma\left[^{\circ}\right]$ & $\begin{array}{c}S A E \\
{\left[\times 1 \mathrm{e}^{-3}\right]}\end{array}$ & $\theta_{0}\left[^{\circ}\right]$ & $\sigma^{-}\left[{ }^{\circ}\right]$ & $\sigma^{+}\left[{ }^{\circ}\right]$ & $\begin{array}{c}S A E \\
{\left[\times 1 \mathrm{e}^{-3}\right]}\end{array}$ \\
\hline Indoor picocell & $2.8 / 2.0$ & $5.9 / 7.1$ & $1.9 / 0.9$ & $2.0 / 2.2$ & $6.9 / 10.5$ & $9.4 / 10.0$ & $0.9 / 1.4$ \\
\hline Outdoor - Indoor & $-0.2 /-0.2$ & $3.9 / 5.8$ & $1.4 / 3.0$ & $-0.2 /-0.2$ & $5.4 / 8.1$ & $5.5 / 8.3$ & $0.5 / 1.6$ \\
\hline \multicolumn{8}{|l|}{ Urban microcell: } \\
\hline $\mathrm{h}_{\mathrm{BS}}=3 \mathrm{~m}$ & $1.4 / 2.0$ & $3.3 / 4.2$ & $1.3 / 2.3$ & $1.6 / 1.4$ & $4.6 / 4.9$ & $4.4 / 7.0$ & $0.7 / 0.2$ \\
\hline $\mathrm{h}_{\mathrm{BS}}=8 \mathrm{~m}$ & $2.0 / 2.2$ & $4.6 / 4.9$ & $0.7 / 0.9$ & $1.8 / 2.0$ & $5.9 / 6.3$ & $6.7 / 7.1$ & $1.0 / 0.6$ \\
\hline $\mathrm{h}_{\mathrm{BS}}=13 \mathrm{~m}$ & $3.4 / 3.6$ & $4.6 / 5.4$ & $1.4 / 2.2$ & $2.0 / 1.8$ & $4.3 / 4.8$ & $8.2 / 10.0$ & $0.4 / 0.2$ \\
\hline Urban macrocell & $5.0 / 5.0$ & $7.6 / 19.7$ & $4.4 / 4.9$ & $2.2 / 2.0$ & $3.9 / 4.6$ & $17.8 / 37.4$ & $0.2 / 0.7$ \\
\hline Highway macrocell & $5.0 / 5.8$ & $4.7 / 6.7$ & $3.4 / 5.0$ & $6.0 / 6.0$ & $8.0 / 9.6$ & $5.7 / 10.0$ & $3.5 / 2.9$ \\
\hline $\begin{array}{l}\text { Average of all } \\
\text { environments }\end{array}$ & $2.6 / 3.6$ & $5.0 / 7.3$ & $1.5 / 2.5$ & $1.6 / 1.8$ & $5.5 / 7.4$ & $8.6 / 13.7$ & $0.2 / 0.6$ \\
\hline
\end{tabular}

that the former yields a better match with the experimental data. The difference is clearest for the cases with largest BS antenna heights (urban macrocell, urban microcell with $h_{\mathrm{BS}}=13 \mathrm{~m}$ ).

In [9], Taga reported considerably larger values for the mean elevation angle and standard deviation of the Gaussian distribution derived from urban macrocell measurements in central Tokyo. However, the urban environment in Tokyo is quite different from that of Helsinki, which could explain the difference. Also the BS antenna height in his measurements was significantly larger $(87 \mathrm{~m})$ than in our measurements $(27$ and $21 \mathrm{~m})$, increasing the portion of power propagated over building rooftops.

\section{Cross-Polarization Power Ratio}

In the case of vertically polarized transmission, the XPR is defined as the power ratio of $\theta$ - and $\phi$-polarized components of the mean incident field. The XPR was obtained from the measurement data as the ratio of the integrals of the mean relative incident power versus elevation angle (see Table I)

$$
\mathrm{XPR}=\frac{\oint P_{\ominus}(\theta) d \theta}{\oint P_{\phi}(\theta) d \theta}
$$

The resulting XPR in each radio environment is shown in Table III. The highest XPR values are obtained in the urban microcell environment; the XPR is approximately $11 \mathrm{~dB}$ for all BS antenna heights. XPR is close to $11 \mathrm{~dB}$ also in the outdoor-indoor case. Instead, for the indoor picocell, urban macrocell, and highway macrocell cases, the XPR is close to $7 \mathrm{~dB}$. In the existing literature, few measurements of the XPR at the mobile station have been reported. In measurements presented in [21], the median "cross-polarization coupling," which is equal to the reciprocal of the XPR, was found to be as high as $-2.5 \mathrm{~dB}$ inside and $-3.5 \mathrm{~dB}$ outside houses in a residential area at $800 \mathrm{MHz}$. The values are clearly lower than what we measured at $2.15 \mathrm{GHz}$. According to measurements by Lee [22] and Taga [9], the XPR in an urban macrocell environment is between 4 and $9 \mathrm{~dB}$ at $900 \mathrm{MHz}$. This is comparable to our measurements at $2.15 \mathrm{GHz}$, although the BS antenna height and BS-MS distance are considerably smaller. According to measurements by Lee and Yeh [22], the differences between the two cross-coupling coefficients $(V P \rightarrow H P, H P \rightarrow V P)$ are less than $2 \mathrm{~dB}$. This indicates that XPR at the mobile station is comparable to the XPR at the base station. In [23], the mean XPR at the base station was found to be $7 \mathrm{~dB}$ in an urban macrocell at $463 \mathrm{MHz}$ when a vertically polarized antenna was used at the mobile station.

In our urban microcell and outdoor-indoor measurements, the range was considerably smaller than in the above references. This would explain the higher obtained XPR values, under the assumption that the number of depolarizing reflections and diffractions on the propagation path increases when the separation between the transmitter and receiver increases. In [24], XPR at the base station was found to be close to $10 \mathrm{~dB}$ in an urban environment at $1800 \mathrm{MHz}$, and building penetration had minor influence on the XPR. In the highway macrocell measurements, the close scattering from the bodywork of the car most probably decreases the XPR. Also in the indoor picocell measurements, the low XPR value can be explained by the high number of close-proximity scatterers around the mobile.

\section{MEG COMPARISON OF HANDSET ANTENNAS}

\section{A. Evaluated Antenna Configurations}

To evaluate MEG as a parameter describing the handset antenna performance, we picked three typical handset antennas: 
a commercial GSM1800 handset with an external meandered monopole antenna and simulated meandered monopole (MEMO) and planar inverted patch (PIFA) antennas attached to a handset model. In addition, we took an omnidirectional discone antenna for reference. We measured the 3-D gain patterns of the discone antenna and the GSM1800 handset in an anechoic chamber with a grid of $10^{\circ}$ in both elevation and azimuth. The measurement frequency was $2154 \mathrm{MHz}$ for the discone and $1747 \mathrm{MHz}$ for the GSM1800 handset. Although the signal distributions were obtained from measurements at 2154-MHz carrier frequency, they can be assumed valid also at $1800-\mathrm{MHz}$ frequency range; the frequency difference is so small that the same propagation mechanisms are effective for waves at both frequencies. The discone was measured in free space only, while the GSM1800 handset was measured both in free space and beside a model of a human head and shoulders. In free space, the handset was oriented vertically, but when placed beside the model, it was tilted $60^{\circ}$ from vertical to correspond to a natural usage position. The handset touched the ear of the model. The used model was Torso Phantom V2.0 by Schmidt \& Partner Engineering AG and was filled with brain-simulating liquid.

We simulated the 3-D gain patterns of the MEMO and PIFA by using a commercial finite-difference time-domain (FDTD) program (XFDTD, version 5.1 Bio-Pro by Remcom, Inc.). Both antennas were attached to the top of a metallic chassis acting as the body of a mobile phone. The simulations were performed both in free space and beside a head model. The simulation frequency was $2154 \mathrm{MHz}$. In free space, the phone chassis were oriented vertically. Beside the head, they were oriented according to the intended use position specified by CENELEC [25]. The phone was tilted $74^{\circ}$ from vertical and $10^{\circ}$ from the ear toward the cheek, as described in [26]. A distance of $5 \mathrm{~mm}$ was left between the head and phone chassis, corresponding to the actual position of the metallic chassis of a mobile phone. The used head model was an FDTD mesh with 2.5-mm voxel resolution remeshed from a standard human head and shoulders model obtained from the software provider.

In both the measurements and the simulations, the head model was in upright position and the nose pointed toward positive $y$-axis (see Fig. 1). The patterns were measured and simulated for the handset placed on both the right and left side of the head, i.e., on the positive and negative $x$-axis sides, respectively. The horizontal (xy-plane) and vertical ( $x z$-plane) cuts of the power gain patterns of all antenna configurations are presented in Fig. 6.

\section{B. Mean Effective Gains of Antennas}

We computed the MEGs of all evaluated antenna configurations using $[9,(6)]$. The distribution of the incident power was assumed uniform in azimuth. As the power distribution in elevation and cross-polarization power ratio, we used the data obtained from the experiments and presented in Section IV. Fig. 7 shows the MEG of each antenna configuration in all radio environments, together with the average MEG and the total antenna efficiency. Table $\mathrm{V}$ presents the average MEG, maximum gain, total antenna efficiency, and cross-polarization discrimination (XPD) of each configuration. The cross-polarization discrimination is given by

$$
\mathrm{XPD}=\frac{\int_{0}^{2 \pi} \int_{-\pi / 2}^{\pi / 2} G_{\theta}(\theta, \phi) \cos \theta d \theta d \phi}{\int_{0}^{2 \pi} \int_{-\pi / 2}^{\pi / 2} G_{\phi}(\theta, \phi) \cos \theta d \theta d \phi}
$$

The total antenna efficiency (including the dielectric losses due to the head model) is obtained by

$$
\eta_{\mathrm{tot}}=\frac{1}{4 \pi} \int_{0}^{2 \pi} \int_{-\pi / 2}^{\pi / 2}\left[G_{\Theta}(\theta, \phi)+G_{\phi}(\theta, \phi)\right] \cos \theta d \theta d \phi .
$$

The reference level in the gain measurement of the GSM1800 handset was the nominal maximum transmission power. Note that the MEG of an antenna in an artificial isotropic environment-i.e., for $p_{\ominus}(\theta, \phi)=p_{\phi}(\theta, \phi)=1 / 4 \pi$, XPR $=1$-is equal to the total antenna efficiency divided by two. Based on Table V, no direct connection can be found between the MEG of an antenna configuration and its total efficiency or gain. Furthermore, it turned out that in most cases, the differences in the MEG values could not be predicted directly by analyzing the plane cuts of the radiation pattern. Only clearly negative XPD with dominating $\phi$ polarization predicted low MEG values.

Fig. 7 shows that the differences in MEG are clearly larger between antennas than between radio environments. This is understandable, since in all environments, most of the power is received at small positive elevation angles (see Fig. 4). In effect, the XPR seems to explain most of the environmental dependence of the MEG, as will be presented below. The reference discone has by far the highest MEG: close to $0 \mathrm{dBi}$ in all environments. This is due to its omnidirectional radiation pattern, high efficiency, and high cross-polarization discrimination. It should be noted that also the MEMO and PIFA have high efficiency in free space, but still their MEGs are significantly lower: on the order of $-5 \mathrm{dBi}$.

When placed beside the head model, the total efficiency of the MEMO drops by $5.9 / 4.6 \mathrm{~dB}$, depending on the side of the head $(\mathrm{R} / \mathrm{L})$. At the same time, the average MEG drops by 2.2/4.7 dB. Fig. 6(c) shows that the $\theta$-polarized pattern of the MEMO in free space has a minimum in the horizontal plane. Instead, beside the head, the maximum of the $\theta$-polarized pattern is produced at the horizontal plane. On the right side of the head, $\theta$ polarization dominates, which partly compensates for the decreased efficiency. For MEMO, the difference in MEGs on the two sides of the head is $2.5 \mathrm{~dB}$. For PIFA, the total antenna efficiency drops by 3.1/2.4 $\mathrm{dB}$ when placed beside the head, and the MEG drops on average by 2.7/3.0 dB.

The average MEG of the measured GSM1800 handset increases by $1.3 \mathrm{~dB}$ when the handset is placed on the left side of the head of the human body model, although the total efficiency drops by $3.3 \mathrm{~dB}$ from the free space value. On the right side of the head, the average MEG is $4.5 \mathrm{~dB}$ lower than on the left side, although the total efficiency is $0.3 \mathrm{~dB}$ higher. The maximum gain of the antenna configuration is almost the same on both sides of the head (note that in free space, the gain is lower). The result clearly indicates that maximum gain or total efficiency of 


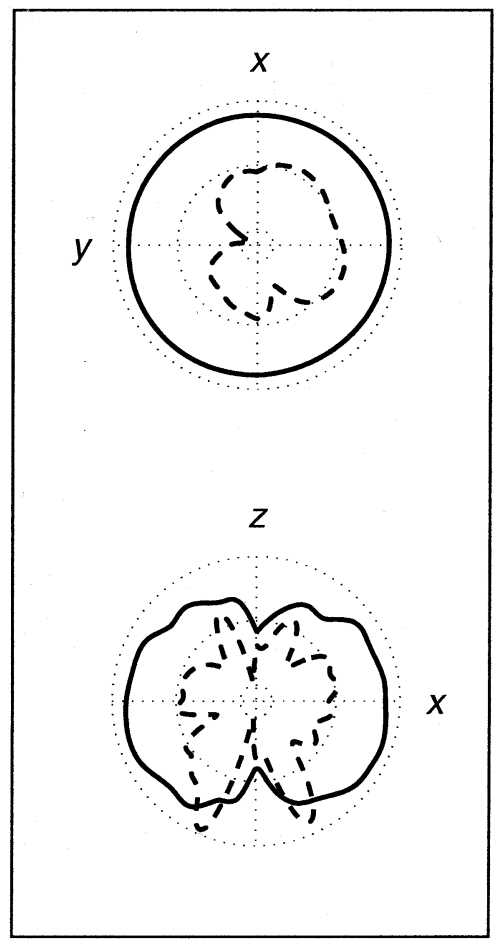

(a) Discone infree space

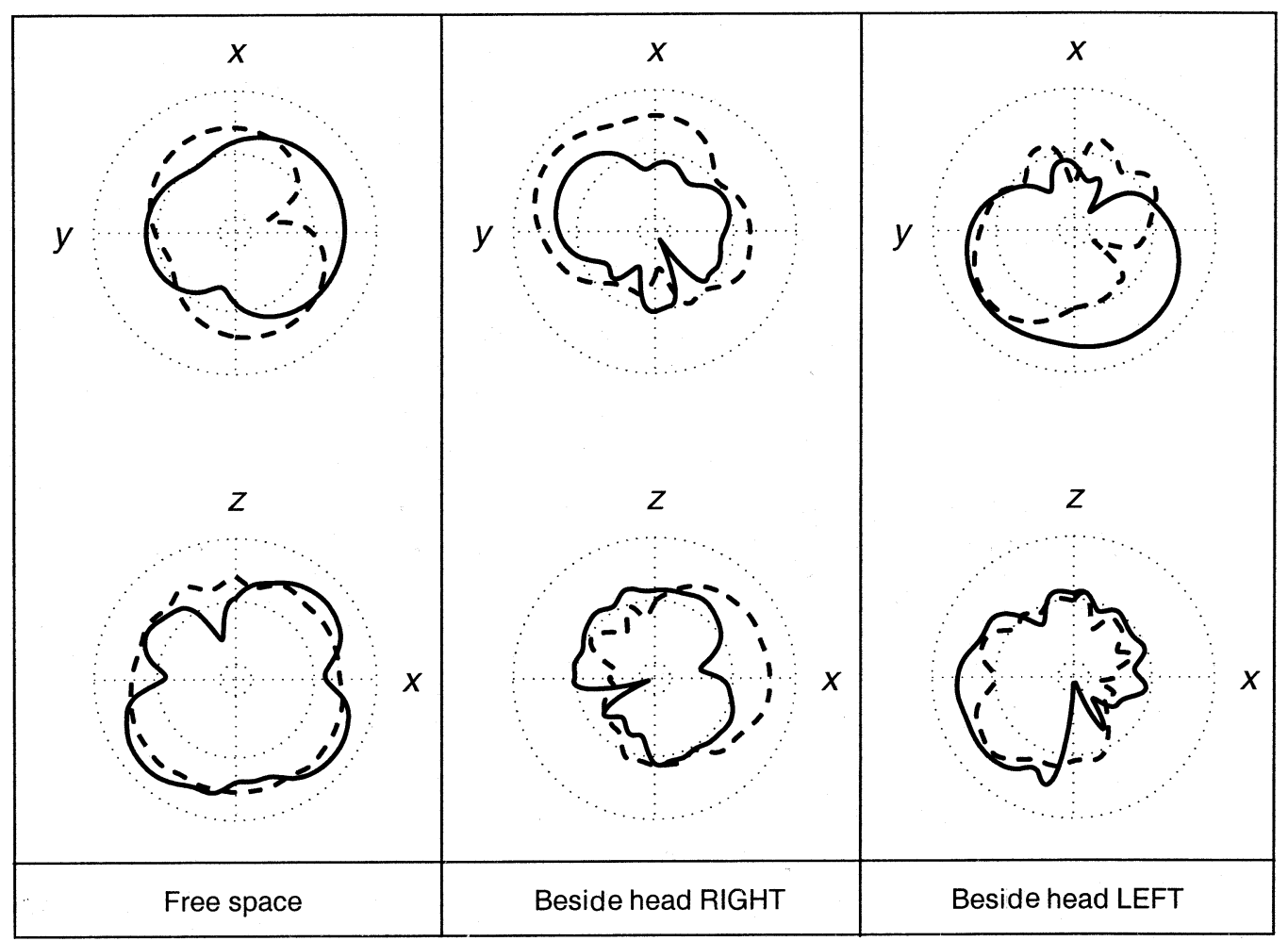

(b) GSM1800

Fig. 6. Gain patterns of evaluated antenna configurations. (a) Horizontal plane cut. (b) Vertical plane cut ( $—-\theta$ polarization, $---\phi$ polarization). The dotted circles correspond to gain levels of $+5,-15$, and $-35 \mathrm{dBi}$.

an antenna is not enough to describe its performance in practical environments.

When any of the two simulated antennas is placed on the left side of the head, the highest MEG values are obtained in environments with lowest XPR (see Fig. 7). The behavior is partly explained by the XPD values of the antenna configurations, which are lower on the left side than the right (see Table V). The opposite happens for the measured GSM1800, which had the antenna located on the opposite corner of the handset. The highest variation of MEG values between different environments is obtained for antenna configurations with negative XPDs. 


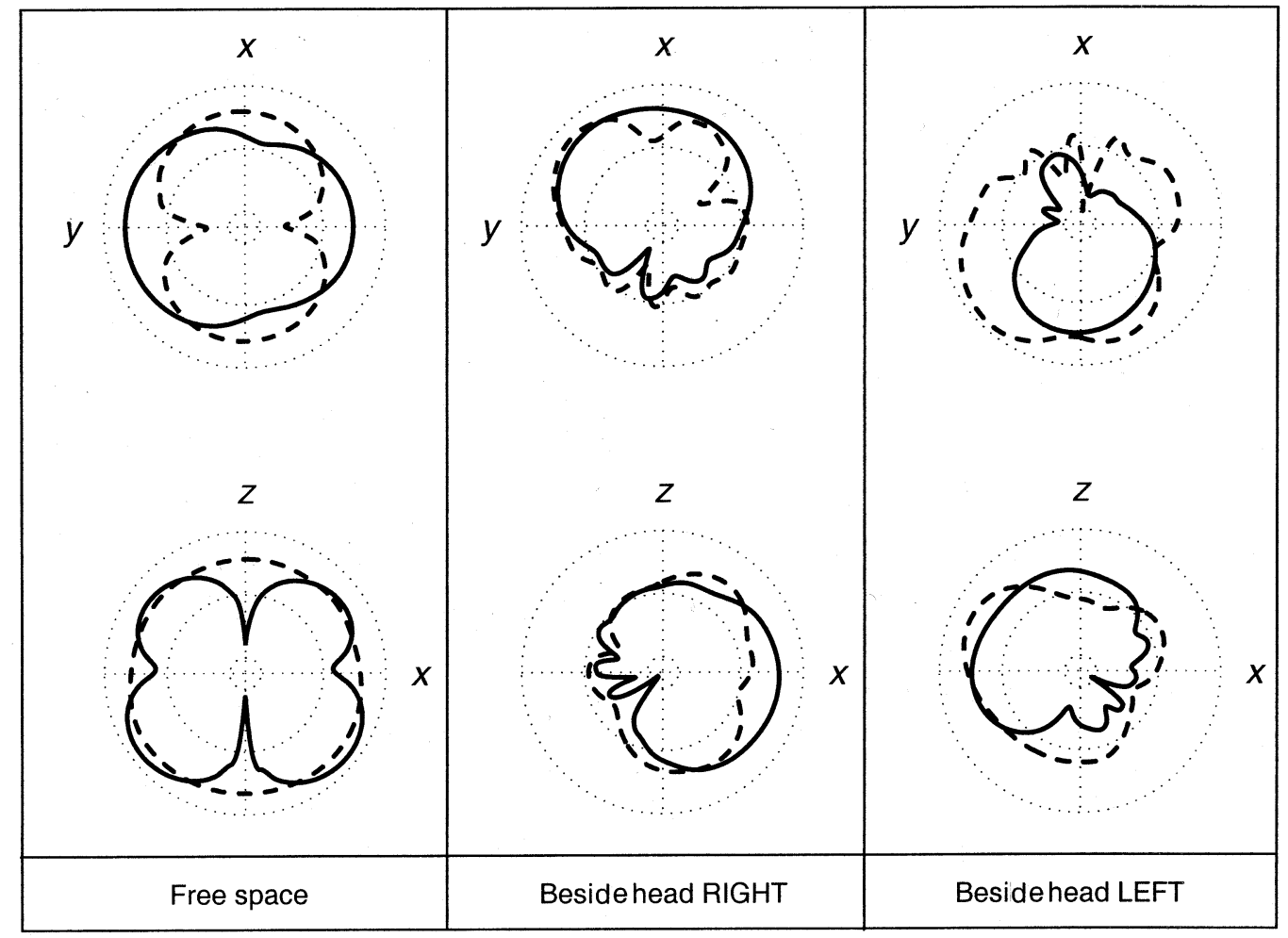

(c) MEMO

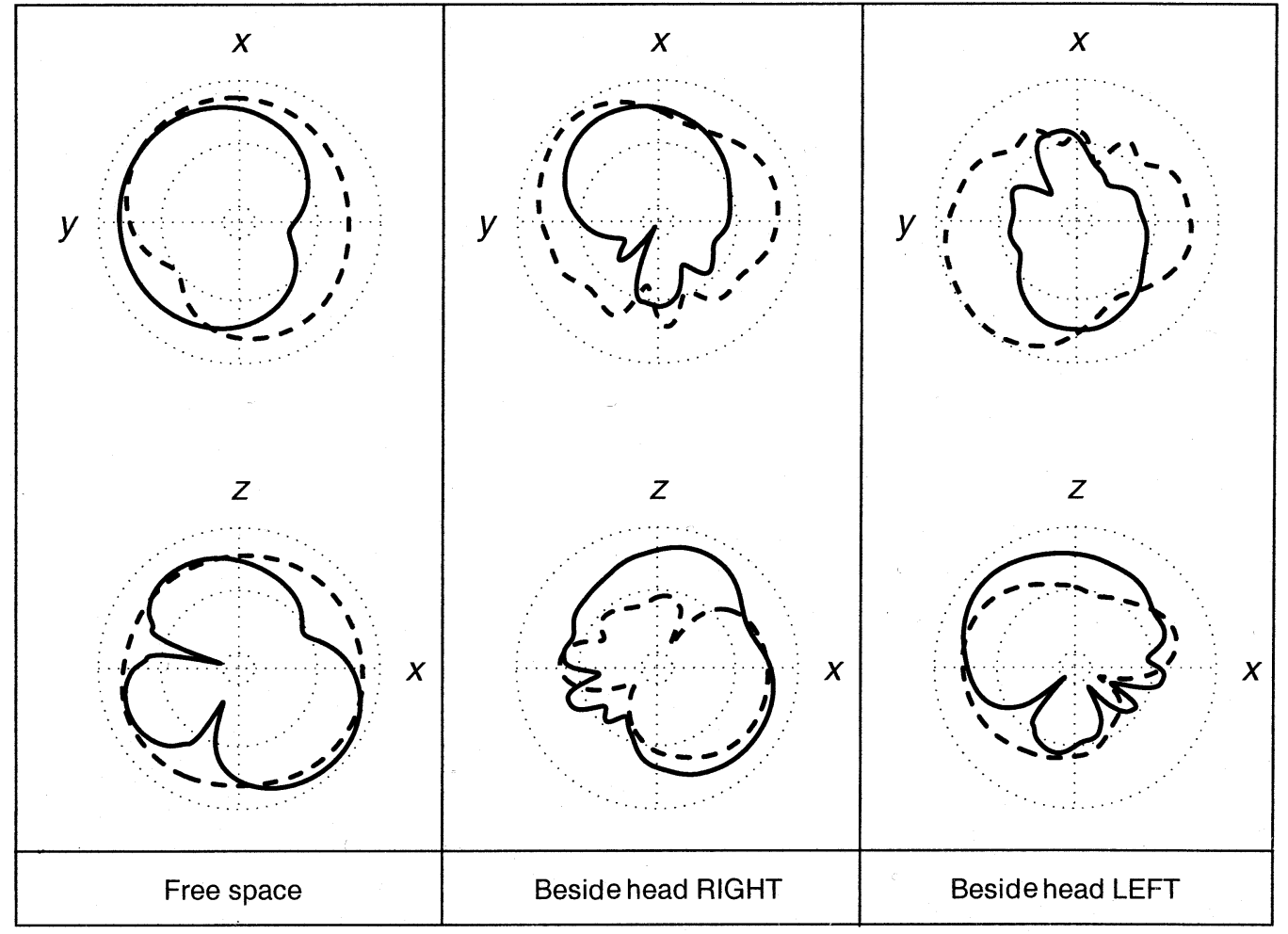

(d) PIFA

Fig. 6. (Continued.) Gain patterns of evaluated antenna configurations. (c) Horizontal plane cut. (d) Vertical plane cut ( $-\theta$ polarization, $---\phi$ polarization). The dotted circles correspond to gain levels of $+5,-15$, and $-35 \mathrm{dBi}$.

It also has been observed previously [27] that the MEG of a handset antenna depends on the side of the head on which the user holds the handset. In [27], the average user influence at $1800-\mathrm{MHz}$ frequency was found to be a loss of $10 \mathrm{~dB}$ for a helical antenna and $3 \mathrm{~dB}$ for a patch antenna. In our analysis, the average decrease in MEG due to the user was 0.8, 3.3, and $2.8 \mathrm{~dB}$ for the measured GSM1800 and the simulated MEMO and PIFA, respectively. However, we only modeled the head of 


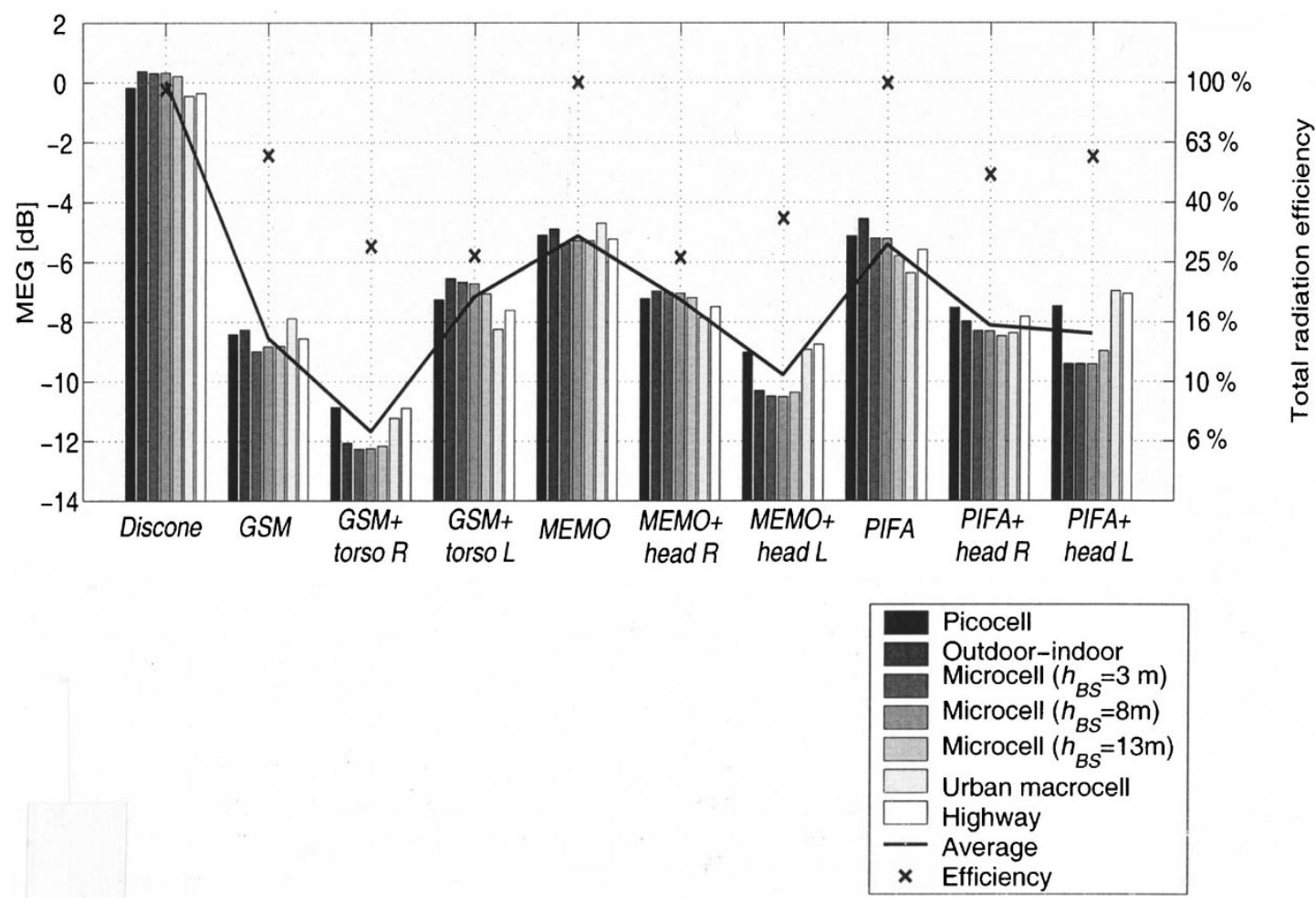

Fig. 7. Mean effective gains of evaluated antennas.

TABLE $\mathrm{V}$

EVALUATED ANTENNA CONFIGURATIONS

\begin{tabular}{|c|c|c|c|c|c|c|}
\hline & Antenna & Tilt angle $^{1}$ & $\eta_{\text {tot }}[\%]$ & $\begin{array}{r}G_{\max } \\
\text { [dBi] }\end{array}$ & $X P D[\mathrm{~dB}]$ & $M E G[\mathrm{dBi}]$ \\
\hline \multirow{4}{*}{ MEASURED } & Discone & $0^{\circ}$ & 95 & 2.9 & 13.0 & 0.0 \\
\hline & GSM1800 & $0^{\circ}$ & 56 & 1.5 & 4.6 & -8.5 \\
\hline & GSM 1800 + torso $\mathrm{R}$ & $60^{\circ}$ & 28 & 3.7 & -5.8 & -11.7 \\
\hline & GSM1800 + torso L & $60^{\circ}$ & 26 & 4.0 & -0.1 & -7.2 \\
\hline \multirow{6}{*}{ SIMULATED } & MEMO & $0^{\circ}$ & 100 & 3.8 & -0.7 & -5.1 \\
\hline & MEMO+head R & $74^{\circ}$ & 26 & 2.8 & 0.2 & -7.3 \\
\hline & MEMO+head L & $74^{\circ}$ & 35 & 3.3 & -5.1 & -9.8 \\
\hline & PIFA & $0^{\circ}$ & 100 & 4.6 & -0.2 & -5.4 \\
\hline & PIFA+head R & $74^{\circ}$ & 49 & 4.3 & -3.0 & -8.1 \\
\hline & PIFA+head L & $74^{\circ}$ & 57 & 4.7 & -5.6 & -8.4 \\
\hline
\end{tabular}

the user (also the shoulders for the measured handset) and not the hand or full body, which partly explains the difference.

\section{Effect of Model Distribution on $M E G$}

To evaluate the two models for the elevation power distribution (see Section IV-C), we repeated the MEG computation for the same antennas using the model distributions, fitted separately for each environment using parameters given in Table IV.
For XPR, the values obtained directly from measurements (presented in Table III) were used. The average differences between the MEGs obtained for the measured and the two modeled EPDs are plotted in Figs. 8 and 9. The MEG errors of both models are small: less than $0.5 \mathrm{~dB}$ for the Gaussian distribution and less than $0.1 \mathrm{~dB}$ for the general double exponential distribution. The differences between the antennas (Fig. 8) are larger than the differences between the environments (Fig. 9). This can be under- 


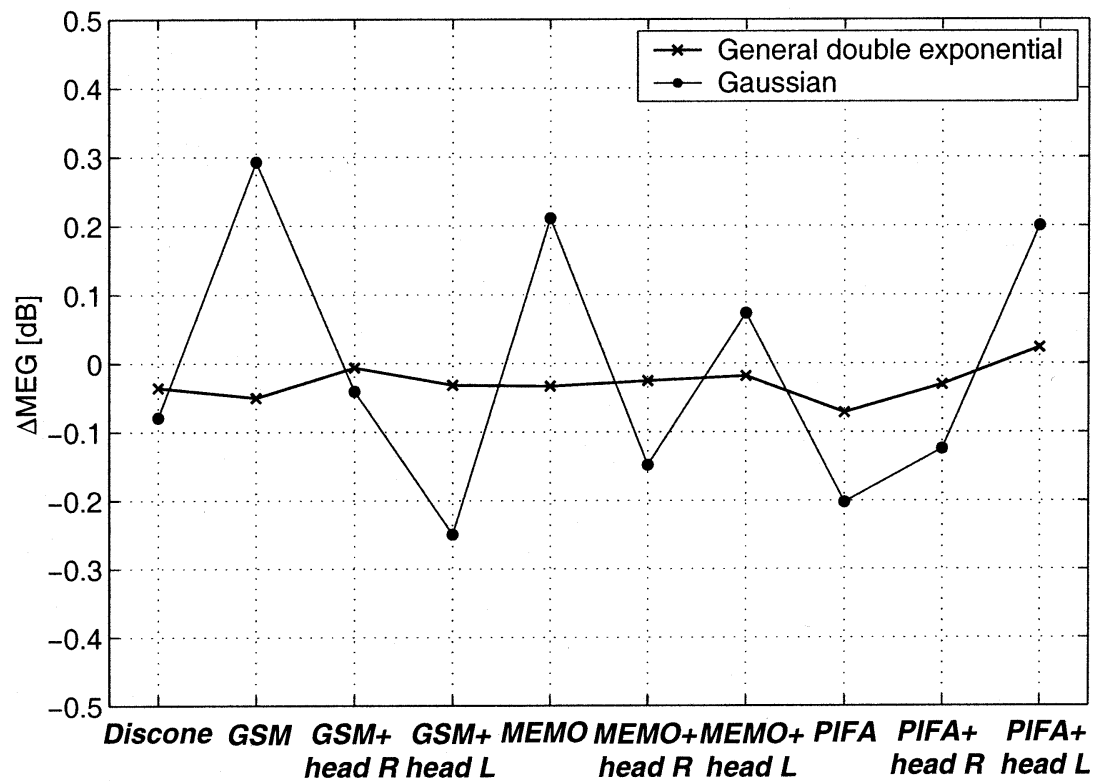

Fig. 8. Difference of MEGs computed from measured and modeled EPDs. Average over environments.

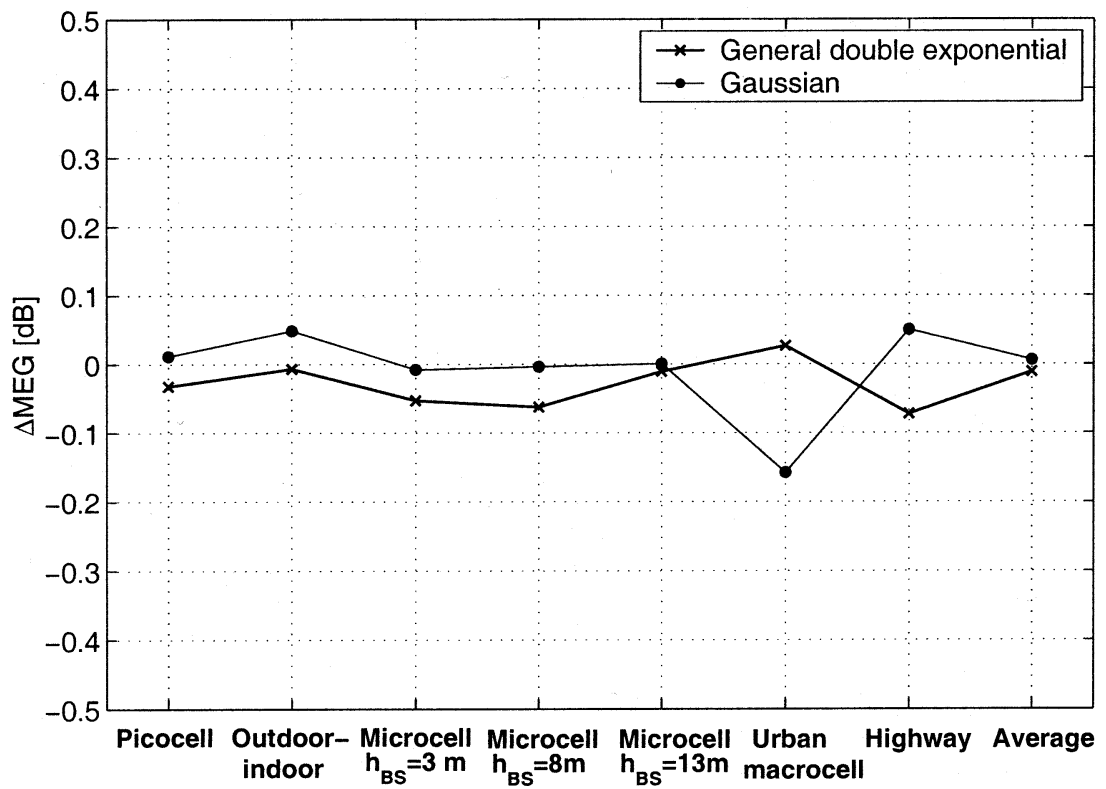

Fig. 9. Difference of MEGs computed from measured and modeled EPDs. Average over antennas.

stood based on the results in the previous section, showing that the environment type has a quite small effect on the obtained MEG.

\section{CONCLUSIONS}

We applied a novel technique for measuring the angular distribution and cross-polarization ratio of the incident power at the mobile station in different types of propagation environments. This information is needed in the evaluation of mobile handset antenna performance in realistic operating environments. The results show that in NLOS situations, the power distribution in elevation has the shape of a double-sided exponential function, with different slopes on the negative and positive sides of the peak. The slopes and the peak elevation angle depend on the environment and base-station antenna height. We noticed that the distribution becomes asymmetrical when the antenna is raised above the rooftop level in an urban environment. With lower BS antenna heights, the power is concentrated only slightly above the horizontal plane.

The measured cross-polarization power ratio was smallest in indoor picocell and urban macrocell environments, on the order of $7 \mathrm{~dB}$. A similar value was obtained also when the mobile antenna was placed inside a car in measurements on a highway. In urban microcell and outdoor-indoor measurements with relatively short ranges, the XPR was fairly large: approximately $11 \mathrm{~dB}$.

We applied the experimental data for analysis of the mean effective gain of several practical handset antennas. The MEG values varied from approximately $-5 \mathrm{dBi}$ in free space to less than $-11 \mathrm{dBi}$ beside the head model. These values are consid- 
erably lower than the $0 \mathrm{dBi}$ typically used in system specifications, e.g., [28]. The result shows that considering only the maximum gain or total efficiency of the antenna is not enough to describe its performance in practical operating conditions. In all measured environments, the cross-polarization coupling was fairly small, indicating that the polarization of a handset antenna should be matched to that of the BS antenna to obtain best performance. However, the polarization of the antenna is sensitive to the usage position of the handset, which should be considered in antenna design.

For most antennas, the environment type has little effect on the MEG, but clear differences exist between antennas. The MEG also depends on the side of the head on which the user holds the handset. Errors in MEG values caused by using any of the two power distribution models instead of the measured power distributions were small: on average less than $0.5 \mathrm{~dB}$ for the Gaussian distribution and less than $0.1 \mathrm{~dB}$ for the general double exponential distribution.

In this paper, we only considered the mean value of the effective gain of a handset antenna. However, it is also important to know the probability levels of which a certain portion of the total incident power is received. The measurements described in this paper allow the analysis of such instantaneous reception efficiency of a handset, as well as more sophisticated analysis like estimation of polarization diversity gain, which are important issues for further studies.

\section{ACKNOWLEDGMENT}

The authors wish to thank M. Toikka, L. Vuokko, J. Ollikainen, P. Suvikunnas, and E. Rinne for their help in carrying out the radio channel measurements.

\section{REFERENCES}

[1] J. B. Andersen and F. Hansen, "Antennas for VHF/UHF personal radio: A theoretical and experimental study of characteristics and performance," IEEE Trans. Veh. Technol., vol. VT-26, pp. 349-357, Nov. 1977.

[2] H. Arai, N. Igi, and H. Hanaoka, "Antenna-gain measurement of handheld terminals at $900 \mathrm{MHz}$," IEEE Trans. Veh. Technol., vol. 46, pp. 537-543, Aug. 1997.

[3] I. Z. Kovács, P. C. F. Eggers, and K. Olesen, "Comparison of mean effective gains of different DCS1800 handset antennas in urban and suburban environments," in Proc. IEEE 48th Vehicular Technology Conf. (VTC98), Ottawa, ON, Canada, May 18-21, 1998, pp. 1974-1978.

[4] K. Sulonen, K. Kalliola, and P. Vainikainen, "Comparison of evaluation methods for mobile handset antennas," in Proc. Millennium Conf. Antennas \& Propagation (AP2000), Davos, Switzerland, Apr. 9-14, 2000, paper 0870.pdf.

[5] B. M. Green and M. A. Jensen, "Diversity performance of dual-antenna handsets near operator tissue," IEEE Trans. Veh. Technol., vol. 48, pp. 1017-1024, July 2000.

[6] G. F. Pedersen and J. B. Andersen, "Handset antennas for mobile communications: Integration, diversity, and performance," in Review Radio Science 1996-1999, W. Stone, Ed. Oxford, U.K.: Oxford Univ. Press, 1999, ch. 5, pp. 970-970.

[7] H. Arai, Measurement of Mobile Antenna Systems. Boston, MA: Artech House, 2001, p. 214.

[8] B. G. H. Olsson, "Simplistic field distribution estimation of a scattered field measurement room," in Proc. IEEE 51st Vehicular Technology Conf. (VTC2000-Spring), vol. 3, Tokyo, Japan, May 15-18, 2000, pp. $2482-2486$.

[9] T. Taga, "Analysis for mean effective gain of mobile antennas in land mobile radio environments," IEEE Trans. Veh. Technol., vol. 39, pp. 117-131, May 1990.
[10] M. B. Knudsen, G. F. Pedersen, B. G. H. Olsson, K. Olesen, and S.-Å. A. Larsson, "Validation of handset antenna test methods," in Proc. IEEE 52nd Vehicular Technology Conf. (VTC2000-Fall), vol. 4, Boston, MA, Sept. 24-28, 2000, pp. 1669-1676.

[11] A. Kuchar, J.-P. Rossi, and E. Bonek, "Directional macro-cell channel characterization from urban measurements," IEEE Trans. Antennas Propagat., vol. 48, pp. 137-146, Feb. 2000.

[12] H. Laitinen, K. Kalliola, and P. Vainikainen, "Angular signal distribution and cross-polarization power ratio seen by a mobile receiver at 2.15 GHz," in Proc. Millennium Conf. Antennas \& Propagation (AP2000), Davos, Switzerland, Apr. 9-14, 2000, paper p1133.pdf.

[13] S. Qu and T. Yeap, "A three-dimensional scattering model for fading channels in land mobile environment," IEEE Trans. Veh. Technol., vol. 48, pp. 765-781, May 1999.

[14] K. Kalliola, H. Laitinen, L. I. Vaskelainen, and P. Vainikainen, "Real-time 3D spatial-temporal dual-polarized measurement of wideband radio channel at mobile station," IEEE Trans. Instrum. Meas., vol. 49, pp. 439-448, Apr. 2000.

[15] R. H. Clarke, "3-D mobile radio channel statistics," IEEE Trans. Veh. Technol., vol. 46, pp. 798-799, Aug. 1997.

[16] _ _ "A statistical theory of mobile radio reception," Bell. Syst. Tech $J .$, vol. 47, pp. 957-1000, July 1968 .

[17] T. Aulin, "A modified model for the fading signal at a mobile radio channel," IEEE Trans. Veh. Technol., vol. 28, no. VT-3, pp. 182-203, Aug. 1979.

[18] J. Kivinen, T. Korhonen, P. Aikio, R. Gruber, P. Vainikainen, and S.-G Häggman, "Wideband radio channel measurement system at $2 \mathrm{GHz}$," IEEE Trans. Instrum. Meas., vol. 48, pp. 39-44, Feb. 1999.

[19] J. Laurila, K. Kalliola, M. Toeltsch, K. Hugl, P. Vainikainen, and E. Bonek, "Wideband 3-D characterization of mobile radio channels in urban environment," IEEE Trans. Antennas Propagat., vol. 50, pp. 233-243, Feb. 2002.

[20] W. C.-Y. Lee and R. H. Brandt, "The elevation angle of mobile radio signal arrival," IEEE Trans. Commun., vol. COM-21, pp. 1194-1197, Nov. 1973.

[21] D. C. Cox, R. R. Murray, H. W. Arnold, A. W. Norris, and M. F. Wazowicz, "Cross-polarization coupling measured for $800 \mathrm{MHz}$ radio transmission in and around houses and large buildings," IEEE Trans. Antennas Propagat., vol. 34, pp. 83-87, Jan. 1986.

[22] W. C.-Y. Lee and Y. S. Yeh, "Polarization diversity system for mobile radio," IEEE Trans. Commun., vol. COM-20, pp. 912-923, Oct. 1972.

[23] R. Vaughan, "Polarization diversity in mobile communications," IEEE Trans. Veh. Technol., vol. 39, pp. 177-186, Aug. 1990.

[24] P. C. F. Eggers, I. Z. Kovács, and K. Olesen, "Penetration effects on XPD with GSM1800 handset antennas, relevant for BS polarization diversity for indoor coverage," in Proc. IEEE 48th Vehicular Technology Conf. (VTC98), Ottawa, ON, Canada, May 18-21, 1998, pp. 1959-1963.

[25] "Considerations for the evaluation of human exposure to electromagnetic fields (EMF's) from mobile telecommunication equipment (MTE) in the frequency range $30 \mathrm{MHz}-6 \mathrm{GHz}$,", Brussels, Belgium, Eur. Spec. ES $59005,1998$.

[26] J. T. Rowley and R. B. Waterhouse, "Performance of shorted microstrip patch antennas for mobile communications handsets at $1800 \mathrm{MHz}$," IEEE Trans. Antennas Propagat., vol. 47, pp. 815-822, May 1999.

[27] G. F. Pedersen, J. Ø. Nielsen, K. Olesen, and I. Z. Kovács, "Measured variation in performance of handheld antennas for a large number of test persons," in Proc. IEEE 48th Vehicular Technology Conf. (VTC98), Ottawa, ON, Canada, May 18-21, 1998, pp. 505-509.

[28] "Universal Mobile Telecommunications System (UMTS): Selection procedures for the choice of radio transmission technologies of the UMTS (UMTS 30.03 version 3.2.0),", ETSI Tech. Rep. TR 101112 V3.2.0 (1998-04), 1998.

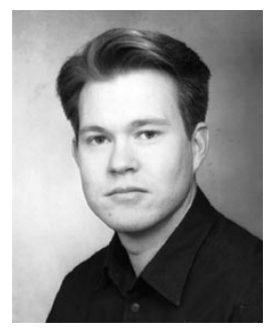

Kimmo Kalliola was born in Helsinki, Finland, in 1972. He received the master of science in technology, licentiate of science in technology, and doctor of science in technology degrees from Helsinki University of Technology (HUT), Helsinki, in 1997, 2000, and 2002, respectively.

From 1997 to 2001, he was a Research Engineer at the Radio Laboratory of HUT developing spatial radio-channel measurement techniques. Since 1999, he has been with the Radio Communications Laboratory, Nokia Research Center. His current research interests are in radio propagation measurements and modeling for wireless communication systems. 


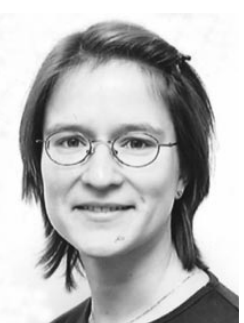

Kati Sulonen was born in Helsinki, Finland, in 1973. She received the master of science in technology and the licentiate of science in technology degrees from Helsinki University of Technology (HUT), Helsinki, in 1999 and 2001, respectively. She is currently pursuing the Ph.D. degree in technology at the Radio Laboratory of HUT.

From 1995 to 1998, she was an Assistant with Technical Maintenance, Information and Communication Networks, Siemens Finland. From 1998 to 1999, she was with the Radio Laboratory of HUT as a Research Assistant. Since 1999, she has been there as a Researcher.

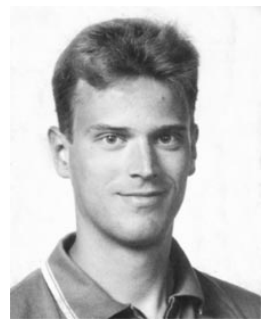

Heikki Laitinen was born in Helsinki, Finland, in 1972. He received the M.S. degree in technology from Helsinki University of Technology, Helsinki, in 1999.

Since 1998, he has been with the Technical Research Centre of Finland, where he is currently a Research Scientist with research interests in radiowave propagation modeling and location techniques.

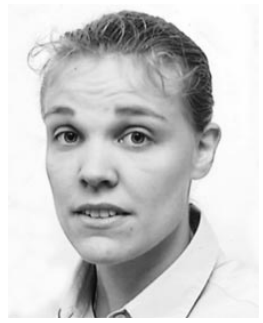

Outi Kivekäs was born in Helsinki, Finland, in 1974. She received the master of science and licentiate of technology degrees in electrical engineering from Helsinki University of Technology (HUT), Helsinki, in 1999 and 2001, respectively. She is currently pursuing the doctoral degree at HUT.

She has been accepted to the national Graduate School in Electronics, Telecommunications and Automation for 1999-2003. Since 1998, she has been a Research Engineer at the Radio Laboratory of HUT, Espoo, Finland. Her research interests include mobile terminal antennas and their user interaction.

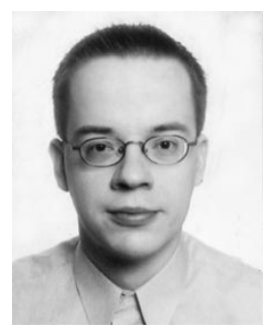

Joonas Krogerus was born in Jämsä, Finland, in 1974. He received the M.S. degree in electrical engineering from Helsinki University of Technology, Helsinki, Finland, in 1999.

Since 1997, he has been with Nokia Research Center, Helsinki, where he is currently a Research Engineer in the Radio Communications Laboratory. His present research interests include mobile terminal antennas and antenna measurement methods.

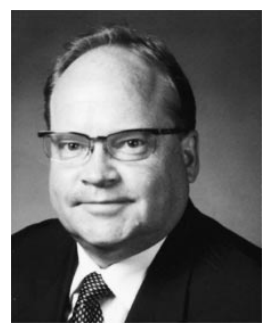

Pertti Vainikainen (M'91) was born in Helsinki, Finland, in 1957. He received the master of science in technology, licentiate of science in technology, and doctor of science in technology degrees from Helsinki University of Technology (HUT), Helsinki, in 1982, 1989, and 1991, respectively.

He was with the Radio Laboratory of HUT from 1981 to 1992 , mainly as a Teaching Assistant and Researcher. From 1992 to 1993, he was Acting Professor of radio engineering, since 1993 Associate Professor of radio engineering, and since 1998 Professor in radio engineering at the Radio Laboratory of HUT. In 1993-1997, he was Director of the Institute of Radio Communications (IRC) of HUT. His main fields of interest are antennas and propagation in radio communications and industrial measurement applications of radio waves. He is the author or coauthor of three books and more than 110 refereed international journal or conference publications. He has received four patents. 